\title{
e-Migrinter
}

$21 \mid 2020$

Famille et migrations

\section{Perte, acquisition, transformations}

Ce que la migration fait à la famille à travers l'exemple d'une famille espagnole originaire de Galice immigrée en France

\section{Emma Rubio-Milet}

\section{OpenEdition}

Journals

Édition électronique

URL : https://journals.openedition.org/e-migrinter/2498

DOI : 10.4000/e-migrinter.2498

ISSN : 1961-9685

Éditeur

UMR 7301 - Migrinter

Référence électronique

Emma Rubio-Milet, «Perte, acquisition, transformations », e-Migrinter [En ligne], 21 | 2020, mis en ligne le 15 janvier 2021, consulté le 20 mai 2021. URL : http://journals.openedition.org/e-migrinter/2498

DOI : https://doi.org/10.4000/e-migrinter.2498

Ce document a été généré automatiquement le 20 mai 2021.

Tous droits réservés 


\title{
Perte, acquisition, transformations
}

\author{
Ce que la migration fait à la famille à travers l'exemple d'une famille \\ espagnole originaire de Galice immigrée en France
}

\author{
Emma Rubio-Milet
}

\section{Introduction : La guerre d'Espagne et son impact sur les populations}

1 La guerre d'Espagne (1936 à 1939) a eu un écho mondial à cause de sa brutalité inédite. Le bombardement par l'aviation allemande de populations civiles et notamment de la petite ville basque de Guernica en avril 1937, a sidéré le monde ; Picasso a immortalisé l'événement, figeant ce nouveau massacre des innocents dans une toile, Guernica, devenue une icône. En janvier 1939, la guerre se termine par la capitulation de la République à Barcelone. C'est la Retirada (la retraite), "[l]es armées franquistes poussent vers le nord des milliers d'Espagnols, femmes, enfants, vieillards, invalides » (Dreyfus-Armand, 1996: p. 38). C'est pourtant un hiver très rigoureux mais des colonnes d'Espagnols prennent le chemin de l'exil, traversent les Pyrénées, fuient la répression du régime de Franco. Pour ces réfugiés en France, "la séparation des familles, quand elles ont pu partir groupées, s'effectue dès la frontière : les femmes, les enfants, et les personnes âgées sont généralement évacués dans divers départements, principalement de l'Ouest et du Centre, vers des centres d'hébergement où ils sont tant bien que mal accueillis; quant aux militaires et aux hommes jeunes, ils sont conduits sous bonne escorte dans des camps de concentration: le terme est employé constamment à l'époque dans les textes et déclarations officiels, au sens où l'on entend " concentrer", afin de les surveiller, ceux que l'on juge "indésirables " (DreyfusArmand, 1996: p. 38). Les familles subissent les premières la violence des guerres civiles ; en Espagne elles sont divisées. Des familles, « célèbres ou anonymes, du nord ou du sud, puissantes ou modestes » comme le montre l'exemple des très célèbres frères Machado, Manuel et Antonio, poètes tous deux reconnus et qui ont combattu chacun dans l'un des deux camps (Moradiellos, 2012 : 16-17). Parfois les familles ont été brisées par les combats, la mort, la répression. Dans ces conditions de violence extrême sur les 
civils, de nombreuses familles républicaines anonymes font le choix de se séparer de leurs enfants et les envoient au Mexique, en Suisse, en Angleterre ou en URSS, les embarquant dans des trains à destination de ces pays lointains où ils ont des contacts et savent que quelqu'un prendra soin d'eux, qu'ils seront mis à l'abri; mais ils ne se reverront plus.

2 Un documentaire intitulé Mémoires en exil (disponible dans ce numéro) illustre cet impact particulier de la guerre d'Espagne sur les familles à travers l'exil forcé des enfants. Modeste dans son ambition mais si juste dans son propos, il a été réalisé par des étudiants de l'université de Bretagne, à partir d'entretiens qu'ils ont eux-mêmes réalisés en Bretagne et à Moscou : « cinq exilés espagnols qui, quatre-vingts ans après, racontent comment, très jeunes, ils furent transférés en France ou en URSS » (Cervantes, 2016) et partagent leurs souvenirs.

3 Ce documentaire témoigne du traumatisme de la séparation familiale. Si des milliers d'enfants furent protégés et sauvés d'un sort funeste, les familles n'en furent pas moins séparées, occasionnant un traumatisme dont l'ethnopsychiatrie des migrations montre la profondeur et la durabilité (Lopez-Pozos, 2001). Réalisé par de jeunes "passeurs » d'une mémoire espagnole de la guerre civile, ce documentaire cherche à faire entendre le traumatisme. Pour certains ou certaines de ces enfants de la guerre, la mémoire trouve à s'exprimer, en particulier quand ils ont bénéficié de l'accueil de familles ou de structures sociales stables qui ont permis qu'un processus de réparation et de légitimation de leur parcours et de leur histoire puisse se réaliser. Il leur reste une fêlure nostalgique mais ils ont pu mener des vies d'une relative normalité. Dans d'autres cas plus extrêmes comme la déportation, la transmission de la mémoire des "vaincus» est trop complexe, reste longtemps indicible, passe par des heurts et des secousses pour les descendants eux-mêmes avant de pouvoir commencer à se dire. Pour celles et ceux qui héritent d'un tel « devoir de mémoire » familiale, la question de sa transmission se pose en termes de fidélité au vécu de proches qui n'ont pu la transmettre eux-mêmes (Martin Domine, 2013).

4 Parallèlement à l'exil des familles républicaines en danger, dès les premières années de l'après-guerre, des migrations dites "économiques » mais considérées comme une conséquence directe de l'événement politique et de la dictature (Rubio, 1977), se produisent à l'intérieur du pays. Conséquences des violences, de la destruction de secteurs entiers de l'activité productive du pays tournée vers l'effort de guerre, de l'épuisement des ressources locales, ou de la disparition des combattants, les migrations économiques sont le fait d'individus qui, pour survivre, décident d'aller trouver ailleurs des ressources que leur région n'est plus en mesure de leur fournir, l'émigration vers l'étranger étant devenue impossible par la fermeture des frontières et l'isolement international de l'Espagne. Bien des Espagnols ont ainsi quitté les territoires périphériques comme la Galice pour rejoindre les régions industrielles de la Catalogne, du Pays Basque, de la région de Madrid ou, dans une moindre mesure, la région de Valence qui accueillaient une main-d'œuvre peu qualifiée et disponible (Barbancho, 1967 ; Canal, 2017).

5 La Galice, dont les institutions s'étaient ralliées aux généraux rebelles dès le lendemain du soulèvement en 1936, n'a connu la guerre civile que sous la forme de la répression et d'une résistance qui tenta de s'organiser jusqu'aux années 1950 en lien avec les territoires voisins des Asturies et du Bierzo Galicia (Yusta, in Martinez, 2017). Aucun front de guerre, aucune bataille n'ont eu lieu sur ce territoire où une " drôle de paix $»^{1}$ 
s'est imposée dans les institutions par la substitution ou l'élimination des fonctionnaires républicains. Mais les privations de l'après-guerre l'ont particulièrement affectée, ce qui a déterminé l'intensification des migrations intérieures.

6 Quelque vingt ans plus tard, lorsque le régime se voit contraint d'abandonner l'autarcie qui a conduit le pays à la ruine économique et sociale (Barciela, 2003 ; Canal, 2017), une nouvelle vague d'émigration internationale se saisit de l'opportunité de la réouverture de la frontière franco-espagnole : des milliers d'Espagnols sans travail et avec de faibles perspectives économiques émigrent vers les pays européens en pleine reconstruction comme la France, la Suisse, l'Allemagne, la Belgique ou le Royaume-Uni, en vagues "spontanées» de "faux touristes» ou par les réseaux officiels (Tur, 2016: 103). D'autres, en nombre bien inférieur cette fois, retrouveront les destinations traditionnelles $\mathrm{du}$ continent sud-américain, réactivant les réseaux solidaires de l'émigration familiale mis en place à l'occasion de la grande vague au tournant des XIXe et XXe siècles (Vicente, 2004).

7 Cette brève contextualisation historique des migrations espagnoles et de leur impact sur les familles à partir d'un événement historique majeur qui a jeté près de 500000 républicains sur les routes de l'exil (Canal, 2017 ; Dreyfus-Armand, 2015) - chiffre qui se stabilise entre 150000 et 200000 après les retours d'une partie d'entre eux pendant les premières années du régime (Rubio, 1977; Dreyfus-Armand, 1996) - et provoqué l'émigration de quelque 400000 migrants économiques en deux vagues migratoires, interne puis externe, qui restent à ce jour les plus importantes au XXe siècle en Espagne et en Europe, permet de mesurer l'impact du conflit sur les populations et sur les familles².

\section{Galice et Catalogne, deux territoires de migrations}




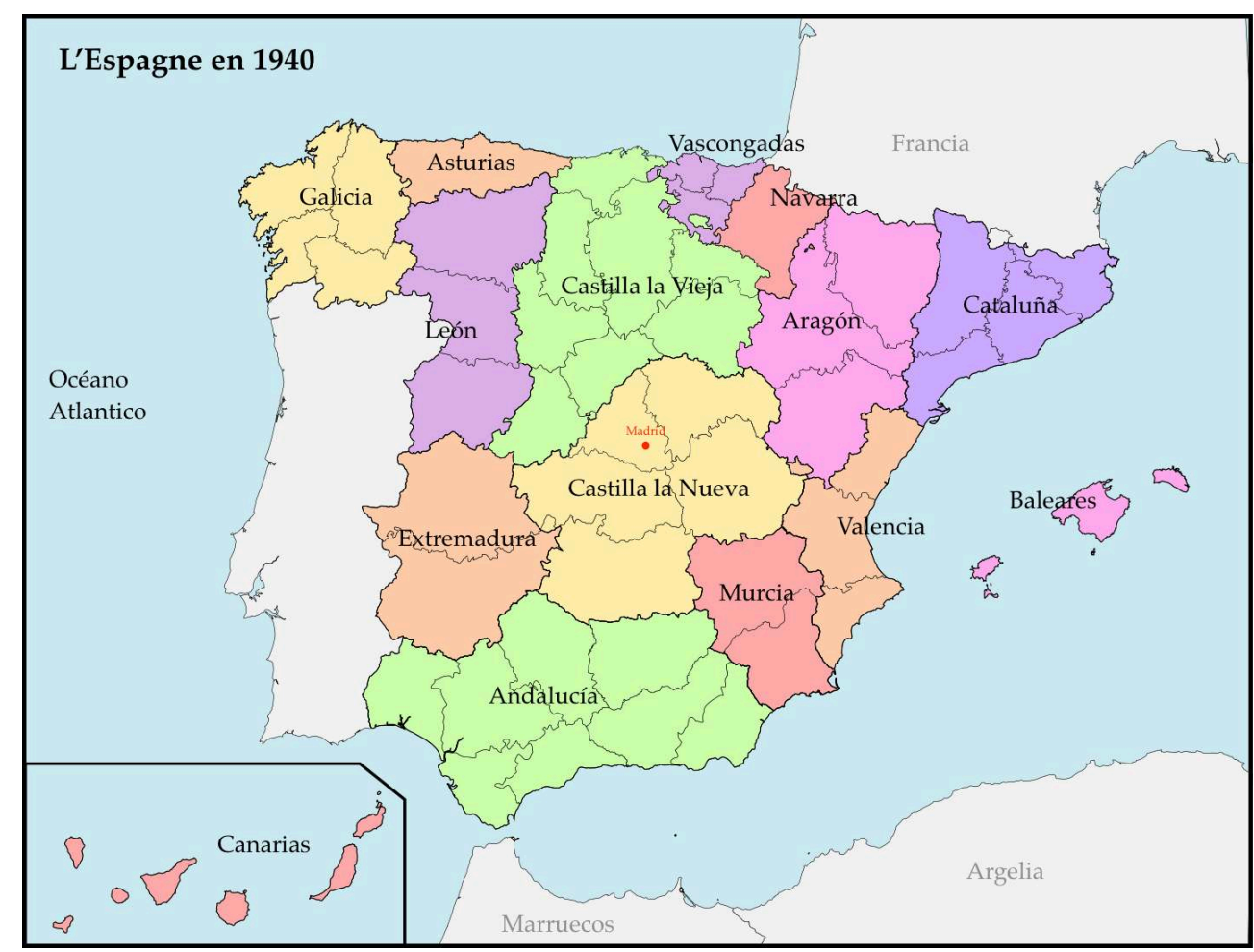

Source : Élaboration de l'auteure (2020)

8 La Galice comme région périphérique à dominante rurale ancestrale avec un faible développement industriel en dehors de l'activité de la pêche et de sa transformation dans les grands ports de A Coruña ou Vigo, a connu dès le XIXe siècle une émigration constante. La Catalogne, à l'autre bout du territoire espagnol sur la façade méditerranéenne, voit au contraire son histoire économique et sociale se développer au rythme d'une industrialisation précoce. La révolution industrielle y produit à partir du XIXe siècle une demande forte de main d'œuvre peu qualifiée pour les usines textiles, la production électrique, etc. Les femmes en particulier vont nourrir dans l'après-guerre les rangs ouvriers où les hommes manquent à une industrie qui se relance, ou apportent leur force de travail dans le secteur des services et des emplois domestiques. Elles sont ces «minyones» (les bonnes), qui remplissent d'une animation nouvelle, le dimanche, les paseos des villes catalanes ${ }^{3}$ où elles vivront, se marieront, la plupart du temps à d'autres immigrés; elles auront des enfants qui seront des Catalans, aux origines diverses, contribuant à renforcer en Catalogne le modèle du "creuset " élaboré par Gérard Noiriel pour la France (Noiriel, 1988).

Comme l'immigration en Catalogne, l'émigration, en Galice, ne commence pas avec le XXe siècle mais constitue au contraire un phénomène continu dès le XVIIe. Le Galicien condense la représentation de l'émigrant espagnol et la Galice devient une terre d'émigration. Ces clichés si répandus suscitent des débats parmi les spécialistes des migrations et de la Galice : l'expérience de l'émigration est-elle l'un des éléments ayant contribué à forger une identité galicienne (Murado, 2008) ou bien cette identité est-elle le fruit d'un discours de l'histoire sociale qu'il s'agit de déconstruire? Nous ne tranchons pas mais constatons que 
«ce lieu commun a occupé la poésie et la prose, la fiction et l'essai, depuis des points de vue différents et avec une intensité variable. Parmi les poètes galiciens, le thème de l'émigration a beaucoup de contributeurs ${ }^{4}$ » (Porrúa, $2012: 403$ ).

Curros Enríquez, Luis Seoane, contemporains célèbres de l'emblématique Rosalía de $\mathrm{Castro}^{5}$, écrivent l'émigration, ses conditions et les sentiments qu'elle provoque. Ils écrivent pour l'émigrant, leur lecteur, et pour ses descendants, transmettant les paysages, les valeurs, les sentiments du pays d'origine, et contribuent à forger une représentation de la situation personnelle du migrant ainsi que «de la situation politique, sociale et économique qui l'a poussé au déracinement ${ }^{6} »$ (Porrúa, 2012 : 404). Une autre figure du galleguismo ${ }^{7}$, Castelao, contribue depuis son exil aux Amériques au maintien et à la diffusion de la culture et de la langue galiciennes par la création de revues très diffusées dans ce que l'on commence à appeler la diaspora galicienne ${ }^{8}$. Le Galicien, el gallego, est devenu l'émigré espagnol par antonomase aux Amériques, particulièrement en Argentine (Murado, 2011 ; Seixas, 2005).

10 Si l'émigration contribue à l'identité galicienne et fait partie de l'imaginaire des Galiciens, elle habite également leur quotidien : pas un, pas une, qui n'ait son histoire d'émigration familiale à raconter. Des figures de bronze à l'effigie de "l'émigrant » (o emigrante), peuplent le territoire de la Galice. Il s'agit la plupart du temps d'un homme en âge de travailler qui attend (le départ ?), toujours flanqué d'une valise réputée en carton, le visage fermé par l'inquiétude ou bien marchant vers son avenir, y projetant l'espoir d'une vie meilleure qu'il fonde sur les nécessités qui le poussent à émigrer autant que sur la culture de l'émigration galicienne. Cette figure devenue universelle, est célébrée par d'innombrables «Festas do emigrante » qui ont toujours lieu sur le territoire galicien ou en Amérique latine.

Figure $n^{\circ} 2$ : Effigie célébrant la 25ème "Festa do Emigrante" en 2008, à Mosteiro, Consello de Pol, Santiago, 2016.

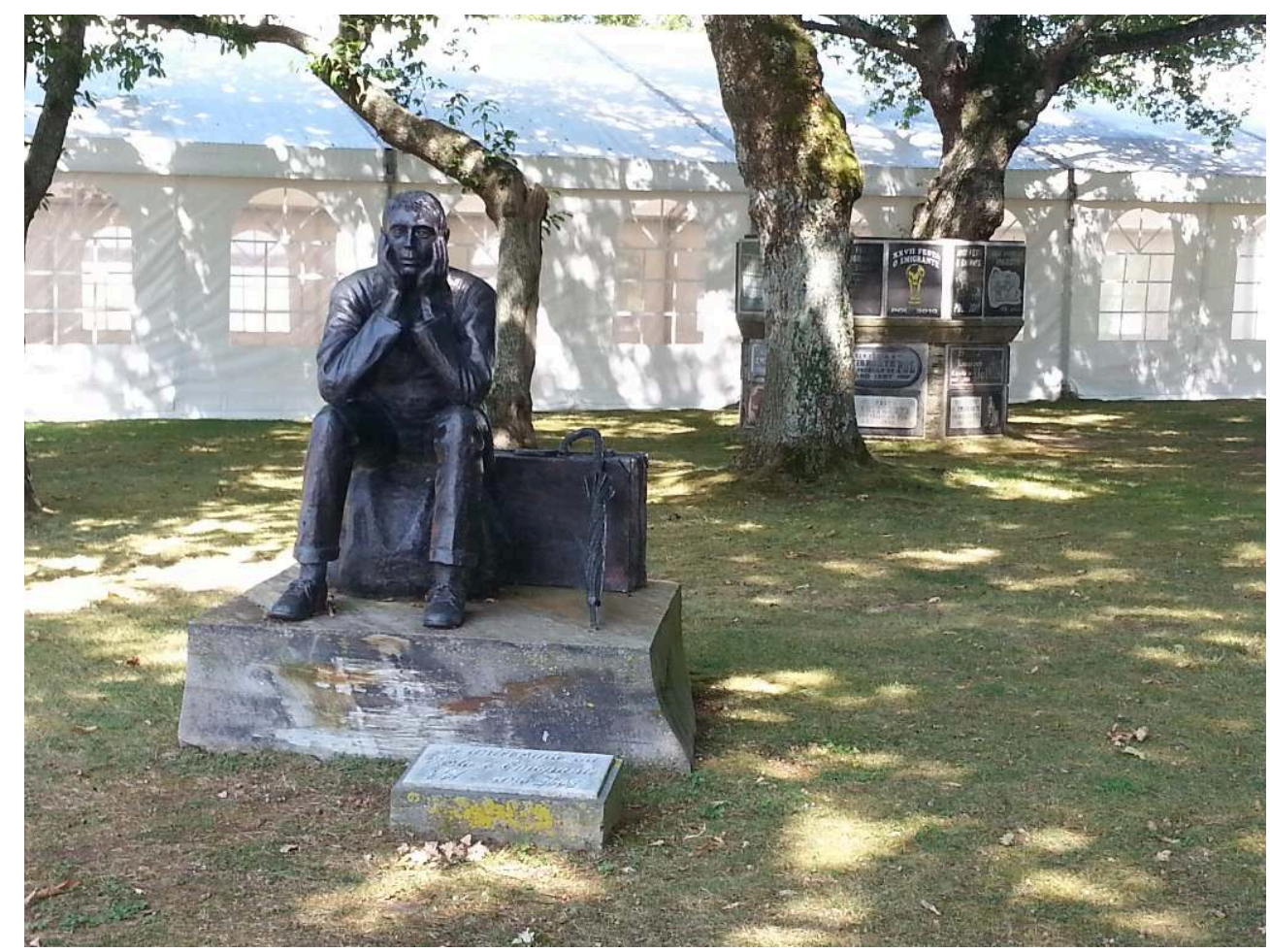

Source : Collection privée. 
Ce cliché figure un homme seul, mais l'émigration galicienne a d'autres visages. Un portrait du photographe galicien Manuel Ferrol immortalise en 1957 une autre représentation de l'émigration galicienne : un homme au visage déchiré par les pleurs tient son fils également en pleurs serré contre lui (Ferrol, 1957).

Figure $\mathrm{n}^{\circ} 3$ : Un père et son fils se séparent d'un familier qui émigre, Port de La Corogne, 1957

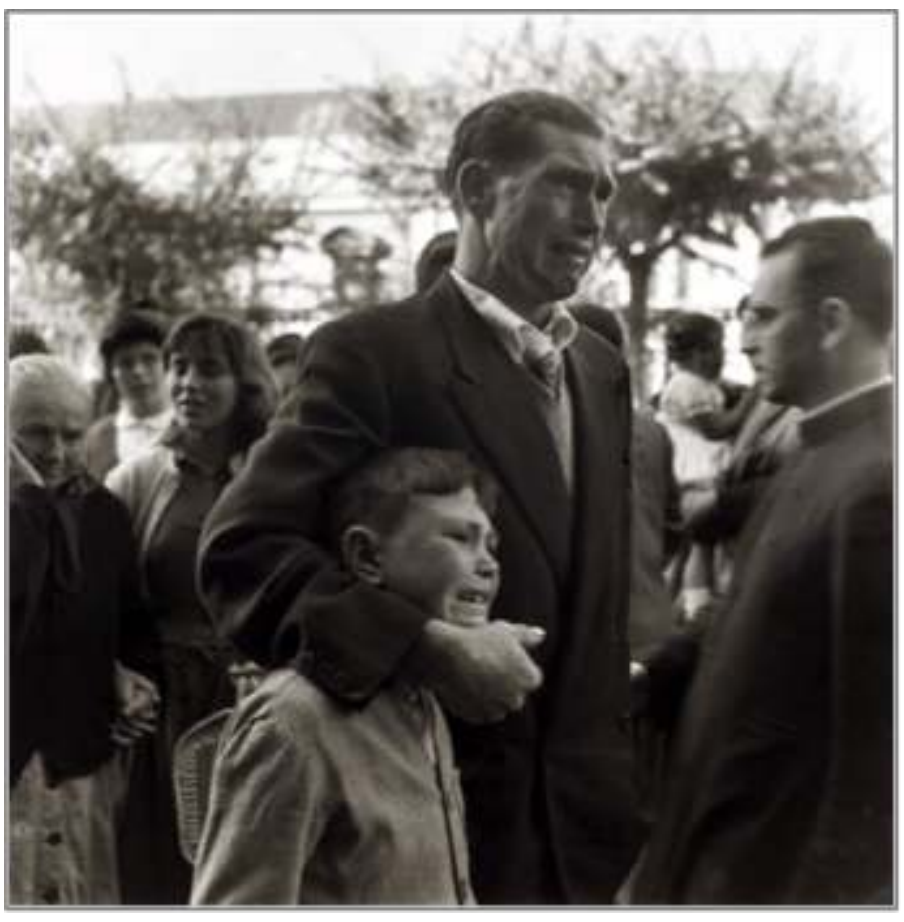

Source : Manuel Ferrol, Archives de Patricia Ferrol

Les circonstances précises de la scène saisie par le photographe sont connues: alors qu'il effectue pour le compte de l'office de l'émigration, un reportage de commande sur les émigrants, en novembre 1957, elle se produit sur le port de A Coruña d'où partent les plus gros bateaux pour l'Amérique. Il s'agit ce jour-là du "Juan de Garay " qui fit traverser l'Atlantique à des milliers de Galiciens; un homme, Xan Calo et son fils Xurxo se séparent d'une partie de la famille, leur mère, une sœur... qui partent pour l'Argentine. La fille de Manuel Ferrol rapporte la réponse de l'enfant questionné plus tard sur ces pleurs poignants : " nous ne pleurions pas parce que nous disions au revoir, nous pleurions parce que nous restions seuls » (Ferrol, 2016). Cette image est devenue une icône universelle de la douleur de la séparation des familles, et la photo galicienne la plus diffusée de tous les temps. Elle répond d'une certaine manière pour nous, dans le silence retentissant de son punctum $^{9}$ (Barthes, 1980), à la question sur la migration et la famille.

13 Cependant, il faut également envisager la question de l'émigration des Galiciens au prisme de l'organisation territoriale et des règles de la transmission de la propriété, soumise à un régime «foral » traditionnel où l'ainé des hommes était l'unique héritier. Cette organisation produit en elle-même un mouvement constant de sa population tout au long de son histoire, accentué par la structure de la famille traditionnellement nombreuse. La pression d'une pauvreté endémique comme conséquence de la crise de 1929 redoublée par les conditions de la guerre d'Espagne vient s'y ajouter et accentue la 
nécessité de l'émigration, l'agriculture de subsistance devenant insuffisante. Malgré tout, comme le démontre Xosé Manoel Núñez Seixas, spécialiste de l'émigration galicienne en Argentine, c'est bien la propriété, pour minuscule qu'elle soit, qui différencie ces enfants de paysans très pauvres d'autres émigrants comme les Andalous par exemple, l'Andalousie étant une autre terre d'émigration historique et pléthorique dans les migrations intérieures espagnoles. Les Andalous émigrent à pied car ils sont des "peones» au service des grands propriétaires et ne possèdent rien, souvent pas même leur habitation ; les Galiciens émigrent en bateau, traversent l'Atlantique, car ils trouvent toujours la possibilité de partir, d'emprunter de quoi faire la traversée (Núñez Seixas, 2005). En Galice, enfin, l'émigration des femmes a toujours été plus importante : dans l'après-guerre comme avant, le sort des filles est décidé par le régime de la transmission de la propriété : elles se marient à des héritiers ou émigrent, mais elles n'héritent pas, sauf dans le cas très rare où elles sont le seul enfant; elles sont celles qui quittent majoritairement les villages où elles sont nées.

On voit que la question des migrations dans l'histoire de la société galicienne se pose éminemment sous l'angle de la famille, traditionnelle et élargie. Par ailleurs, la forme de la propriété (minifundio) et sa transmission à un seul héritier mâle la prédisposent à une émigration conçue comme un mouvement habituel de la vie. La migration impose ses contraintes constantes: celle du départ et la nécessité de s'adapter, si l'on va audelà des régions voisines, à de nouveaux modes de vie, à de nouveaux usages, voire à d'autres cultures et à d'autres langues; dans ce travail nous nous interrogeons sur le devenir dans la migration des liens affectifs qui unissaient les membres familles.

La famille est une réalité complexe et un objet difficile à définir du fait de sa familiarité même pour quiconque souhaite l'aborder (Vallon, 2006). Nous en limiterons le contour à celui du phénomène humain et à l'institution qui prend son origine dans le mariage dont la fonction est biologiquement et socialement la reproduction, un «petit groupe d'êtres » intimement liés les uns aux autres (Zola, 1985) par les liens du sang et qui partagent, le temps de l'enfance des uns (les enfants), et un espace-temps organisé par les autres (les parents). La vocation de cette institution est la transmission: des biens quand il y en a (la fonction sociale de la lignée) mais aussi des valeurs familiales et des normes sociales. Chaque génération sera capable en fonction de ces valeurs communes partagées de les transmettre en partie ou de recréer, dans son propre noyau familial, des valeurs, en lien avec ses propres circonstances de vie. La famille est par définition pour les anthropologues, un groupe humain et une institution sociale dont la variabilité n'a d'égale que la variabilité des sociétés humaines. Elle a « des capacités d'invention et d'évolution, même dans des contextes économiques et sociaux difficiles " (Vallon, $2006: 159)$.

Dans les circonstances de la migration, la transmission est interrompue par la distance géographique et une coupure s'opère. Comment la famille se transforme-t-elle dans des parcours migratoires complexes marqués par des ruptures et des recommencements? Si les départs impliquent des ruptures contingentes de toutes sortes (territoriales, psychiques, affectives, culturelles), comment, la famille se réalise-t-elle dans la réalité des individus migrants et comment sa structure, référée à celle de la famille d'origine, évolue-t-elle? Comment les familles migrantes résolvent-elles la première de ces difficultés, celle de la distance géographique?

17 Ce questionnement sur le devenir de la famille dans la migration, nous proposons d'y répondre en revenant sur le parcours migratoire d'une famille originaire de Galice au 
sein de laquelle la figure du migrant traverse les générations et marque tant la culture locale que le rapport affectif, identitaire et social des individus au territoire. Nous nous appuierons sur le témoignage d'Amélie, qui a fait revivre pour nous sa mémoire d'enfant immigrée en France avec sa famille en 1964, au cœur de la vague d'émigration " économique » amplifiée par la réouverture du régime aux relations internationales et au commerce avec les pays voisins. C'est au cours de travaux de recherche sur le terrain que ce témoignage a surgi, sans que nous ne puissions alors ni l'enregistrer formellement, ni l'exploiter plus avant. Son expérience sensible au sein d'une famille au parcours migratoire en plusieurs étapes et toujours à l'initiative de sa mère, Julia, dont nous explorions le parcours, et surtout le bilan très négatif qu'elle en tire, à près de 50 ans, a provoqué nos interrogations sur ce que la migration fait à la famille.

\section{Un parcours migratoire au long cours}

18 Julia ${ }^{10}$ R.P. est née en 1928 à Fruxil, un village qui compte une quinzaine de familles, dans la Galice intérieure et rurale caractéristique par sa structure foncière et son organisation en innombrables paroisses, héritée du Moyen-Âge :

« Dans la Galice intérieure, l'extrême dispersion de la population, un système de très petites propriétés, les minifundios, (il a fallu attendre 1926 et la dictature de Primo de Rivera pour qu'un décret de rachat des terres abolisse le système de propriété de la terre d'origine féodale) et le nombre très élevé de communes (la Galice concentre $50 \%$ des communes d'Espagne alors qu'elle n'occupe que $6 \% \mathrm{du}$ territoire espagnol) ont contribué à la création - et au maintien dans les zones rurales - d'un espace social dominé par les relations familiales, la tradition, la mutualisation et surtout des relations de type clientéliste (caciquisme) qui ont poussé les Galiciens pauvres et analphabètes à aller chercher fortune ailleurs [...]. L'exode a participé au vieillissement et à la féminisation de la société locale et a accru son immobilisme contrairement à la Catalogne et [au] Pays Basque. Les années de dépression de la République, puis les années d'autarcie de la période franquiste ont accentué le recul économique et social et le caractère rural de la Galice » (Delfour, 2009 : 125-126).

L'Espagne et plus particulièrement la Galice ont subi très durement la crise économique de 1929. La famille de Julia s'est constituée dans la misère des réalités d'alors. Son père, Ramón R.R., avait émigré en Argentine, probablement dans les années 1920. Fiancé à Genoveva P.L. originaire d'un village voisin, il ne pouvait comme il le souhaitait, s'installer dans la maison familiale dont il hérite, bien qu'il ne soit pas l'ainé, après la mort de ses parents auprès desquels il était resté en l'absence de son frère ainé. Les trois années qu'il passe outre-Atlantique lui permettent de réaliser son projet et de reprendre avec Genoveva épousée dès son retour, la ferme familiale et d'y fonder leur famille en achetant, grâce à ses gains, quelques parcelles. Comme Ramón, à cette époque, un grand nombre de Galiciens, essentiellement des hommes, ont émigré en Argentine, la plupart du temps avec la perspective d'un retour que tous n'ont pas réalisé ${ }^{11}$. La fratrie s'était dispersée dans les années 1920 pour combattre la dictature de Primo de Rivera (1923-1931). Pendant la guerre (1936-1939) deux de ses frères, dont l'ainé, ont combattu dans les rangs de la République en Catalogne et l'un d'eux serait mort au combat. L'autre, Julio, est resté en Catalogne et s'y est définitivement installé. Une sœur avait émigré comme domestique dans une région voisine, d'autres frères ne sont pas revenus d'Argentine et les contacts se sont perdus. Julio sera, dans les années 
d'après-guerre, le premier ancrage du réseau d'émigration familial entre Fruxil et Manresa en Catalogne.

Figure $n^{\circ} 4$ : La famille R.P. entre 1938 et 1940

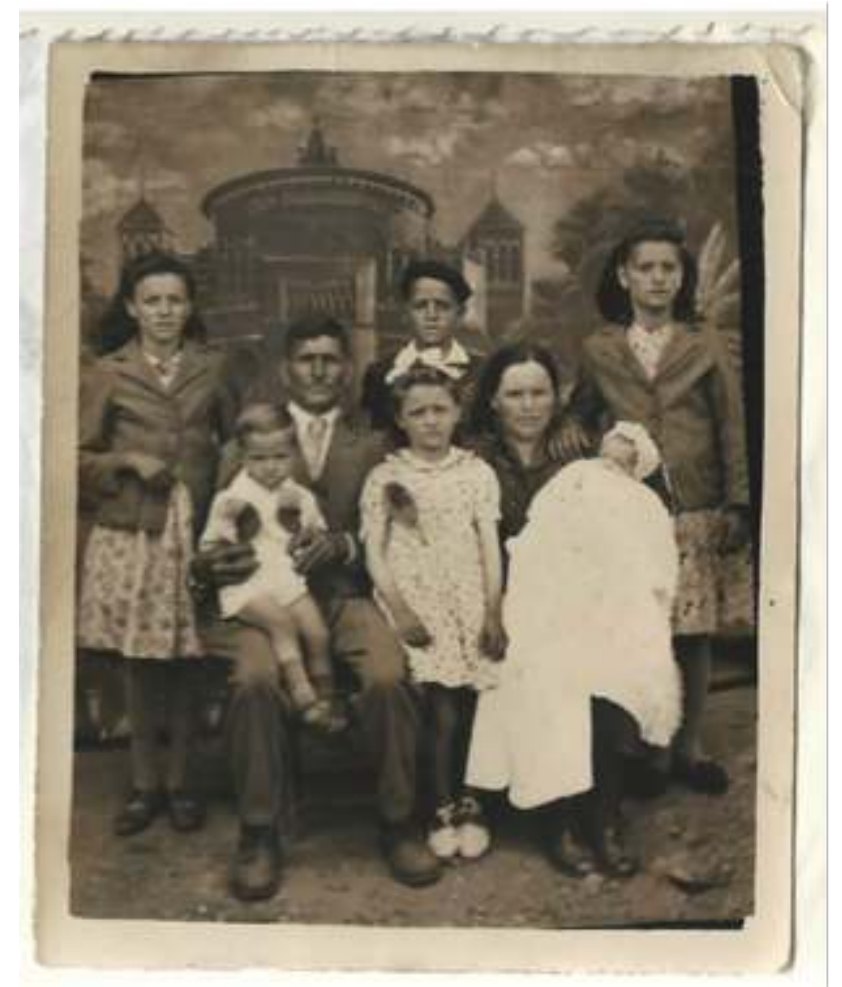

Source : Collection privée.

Dans la famille R.P. Julia est l'ainée d'une fratrie de deux frères et quatre sœurs nés vivants dont l'héritier est José, l'ainé des deux garçons. Dans les années 1940, toutes les nouvelles bouches deviennent difficiles voire impossibles à nourrir. Les filles sont encouragées à partir pour trouver leurs propres moyens de subsistance.

\section{Première étape : de Fruxil à Manresa, un réseau d'entraide familiale}


Figure $n^{\circ} 5$ : Le trajet de Julia

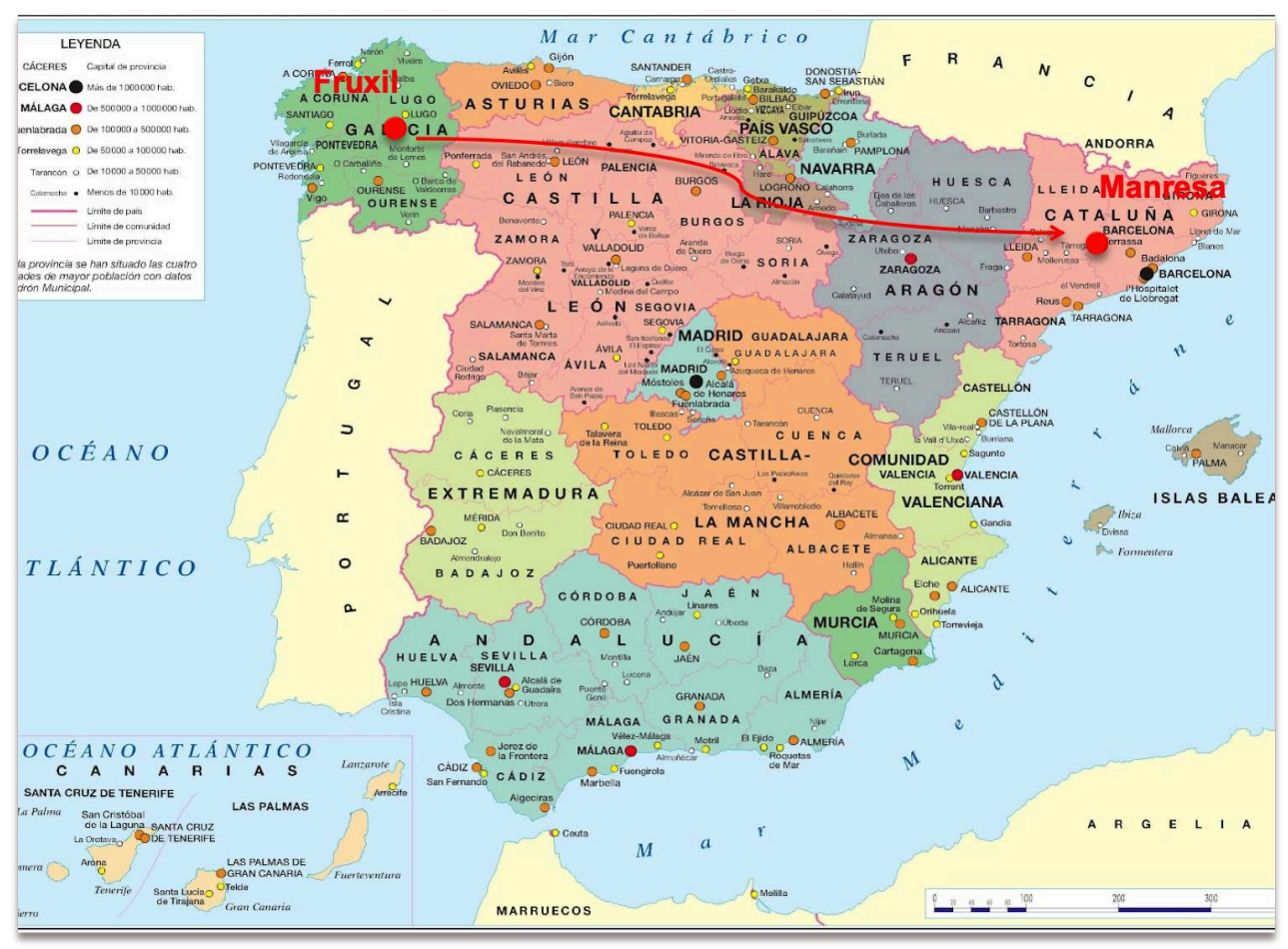

Source : Élaboration par l'auteure (2020)

Vers 1944, Julia R.P., à peine âgée de 16 ans, quitte son village pour Manresa, une ville moyenne industrielle au cœur de la Catalogne où réside son oncle Julio. Elle fait le voyage avec lui et sa tante, profitant de la visite du couple à la famille galicienne. Il est employé à la Centrale électrique (FECSA) et fait figure de pionnier dans l'histoire migratoire de la famille car il connait les possibilités d'embauche dans les usines de la ville $^{12}$. Julia vivra chez lui et c'est par son intermédiaire qu'elle aura, dès son arrivée, un emploi comme ouvrière dans l'une des nombreuses usines textiles de la ville. Cependant, refusant de rendre des comptes à cet oncle qui exige qu'elle lui remette l'intégralité de ses gains, elle cherchera rapidement un emploi logé, - sans le lui dire car il menace de la renvoyer au village. Elle trouvera seule un emploi de "minyona", employée de maison au service d'une famille aisée, dont le père était un opticien de Manresa. Mineure car la majorité est atteinte à vingt-et-un ans, elle doit demander l'autorisation à ses parents, une émancipation qu'elle obtiendra sans difficulté de leur part, malgré la distance. Elle évoque avec enthousiasme ce démarrage actif de sa vie professionnelle où elle partage la charge de travail avec une autre domestique, immigrée d'Andalousie. 


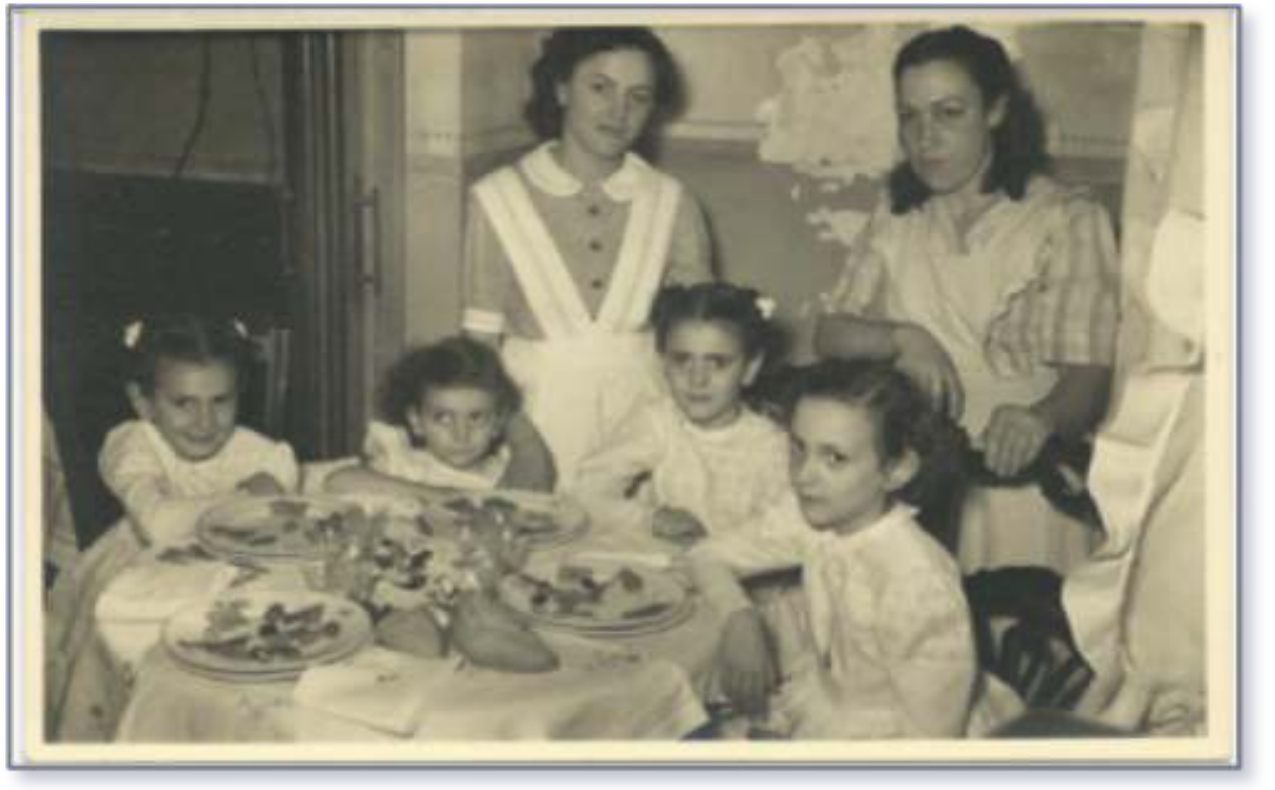

Source : Collection privée.

21 Ses trois sœurs émigreront à leur tour, les unes après les autres, quelques années plus tard et seront généralement ouvrières dans le textile. Son frère cadet, Ramón R.P., bénéficiera lui aussi du réseau de l'oncle pionnier et de sa sœur. Logé chez elle, puis chez leur oncle, il sera tout d'abord ouvrier du textile avant d'être embauché à la Centrale électrique où il fera la totalité de son parcours professionnel ${ }^{13}$.

D'un mode de vie très rural et rude, sans eau courante, sans électricité, sans routes et avec pour seul moyen de transport le dos de l'âne ou la charrette, Julia, ses sœurs et son frère passent à celui, très urbanisé, de la ville industrielle catalane. Cela correspond parfaitement au projet que Julia imaginait :

je voulais partir de Fruxil ! Je devais avoir dix ans que je voulais déjà partir ;

[...] laisser la boue... ce n'était pas goudronné à cette époque-là. Je détestais ces sabots qu'on portait et je voulais partir rien que pour tout ça... ${ }^{14}$.

Grâce au réseau d'émigration familial qui s'élargissait, réseau de solidarité à même d'accueillir les arrivants et de les aider à s'intégrer dans une société en expansion, le projet migratoire de toute la famille R.P. est réussi. D'autres formes d'entraide familiale apparaissent dans le parcours de Julia, ainsi que d'autres épreuves, comme nous allons le voir.

\section{Des Castillans en Catalogne}

Julia rencontre son mari à Manresa, au bal du dimanche, le lieu de sociabilité le plus fréquenté par les jeunes. Immigré de l'intérieur, originaire de Torrejoncillo del Rey, un petit village de la Castille « pouilleuse ${ }^{15}$, dans la province de Cuenca, José R.M. est l'un des nombreux enfants d'une famille pauvre et sans propriété. Les privations de l'aprèsguerre, alors qu'il avait entre cinq et huit ans ont marqué son enfance. Lorsque le curé de la paroisse propose à ses parents de les prendre, lui et son frère, au séminaire de Castellón de la Plana, ils se laissent convaincre par l'espoir qu'ils mangeront à leur 
faim, qu'ils seront éduqués et sortiront de leur condition misérable. Quelques années plus tard, des suspicions de maltraitances amèneront leur frère ainé à demander à leur père l'autorisation d'aller les chercher au séminaire. Les trois frères se retrouveront à Manresa où l'ainé a développé son activité professionnelle dans le bâtiment et ses deux jeunes frères travailleront quelques temps comme ouvriers plâtriers auprès de lui.

Figure $n^{\circ} 7$ : Le trajet de José

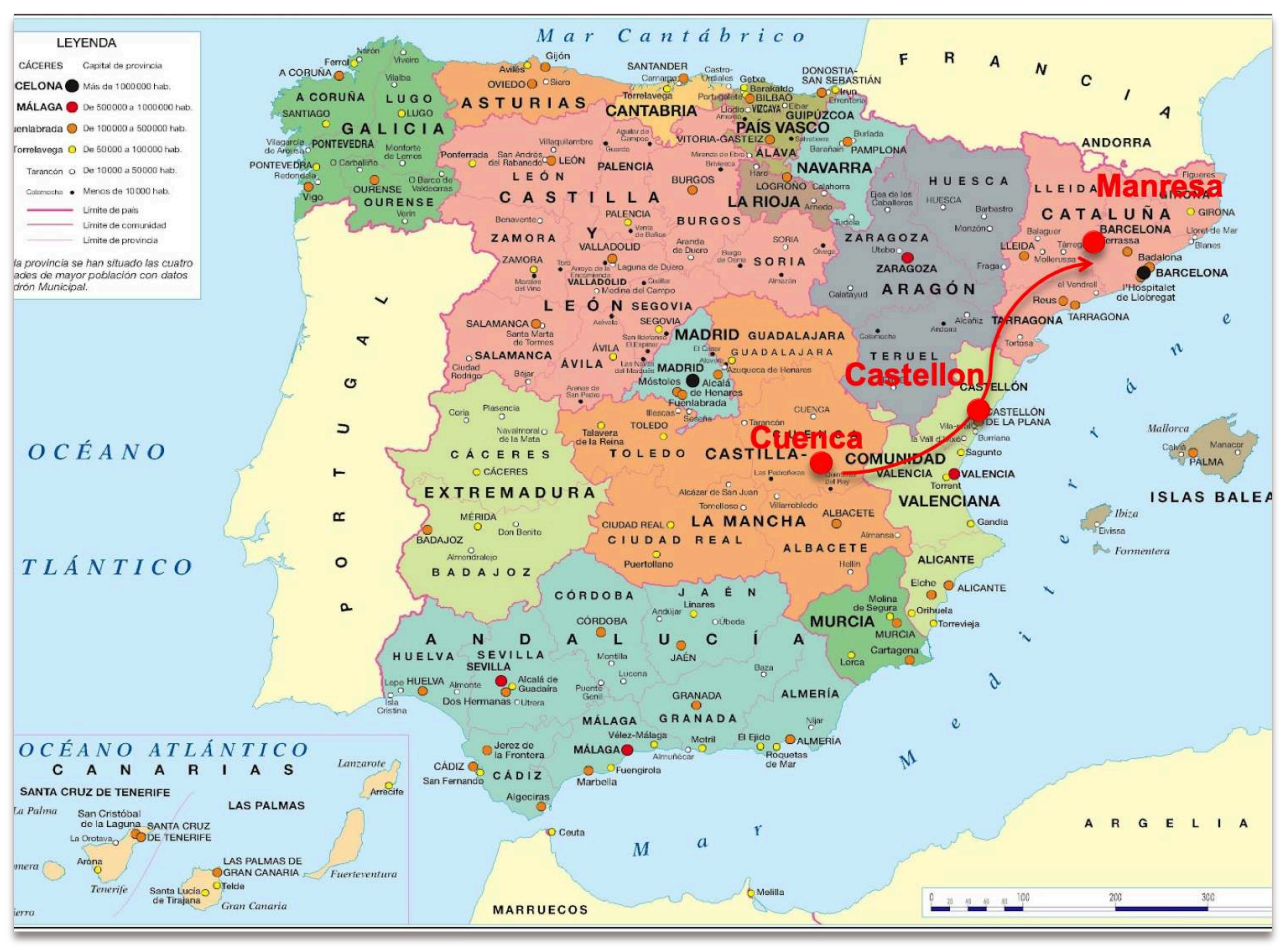

Source : Élaboration par l'auteure (2020).

L'observation de ce moment du parcours de la famille R.M. immigrée de Castille nous permet d'entrevoir un autre schéma de solidarités familiales : passant par la médiation institutionnelle de l'Église - très présente auprès des familles sous le franquisme - et de sa fausse promesse éducative, il se produit un transfert de la responsabilité parentale vers le grand frère qui exerce protection et autorité sur les plus jeunes, qui détecte un danger, obtient de son père l'autorisation de les extraire de l'institution et leur permet, pour un temps, une intégration par le travail dans une activité que lui-même conservera toute sa vie. 
Figure $\mathrm{n}^{\circ} 8$ : Les trois frères R.M., (de gauche à droite : José, Daniel et Carmelo), des Castillans à Manresa en 1950

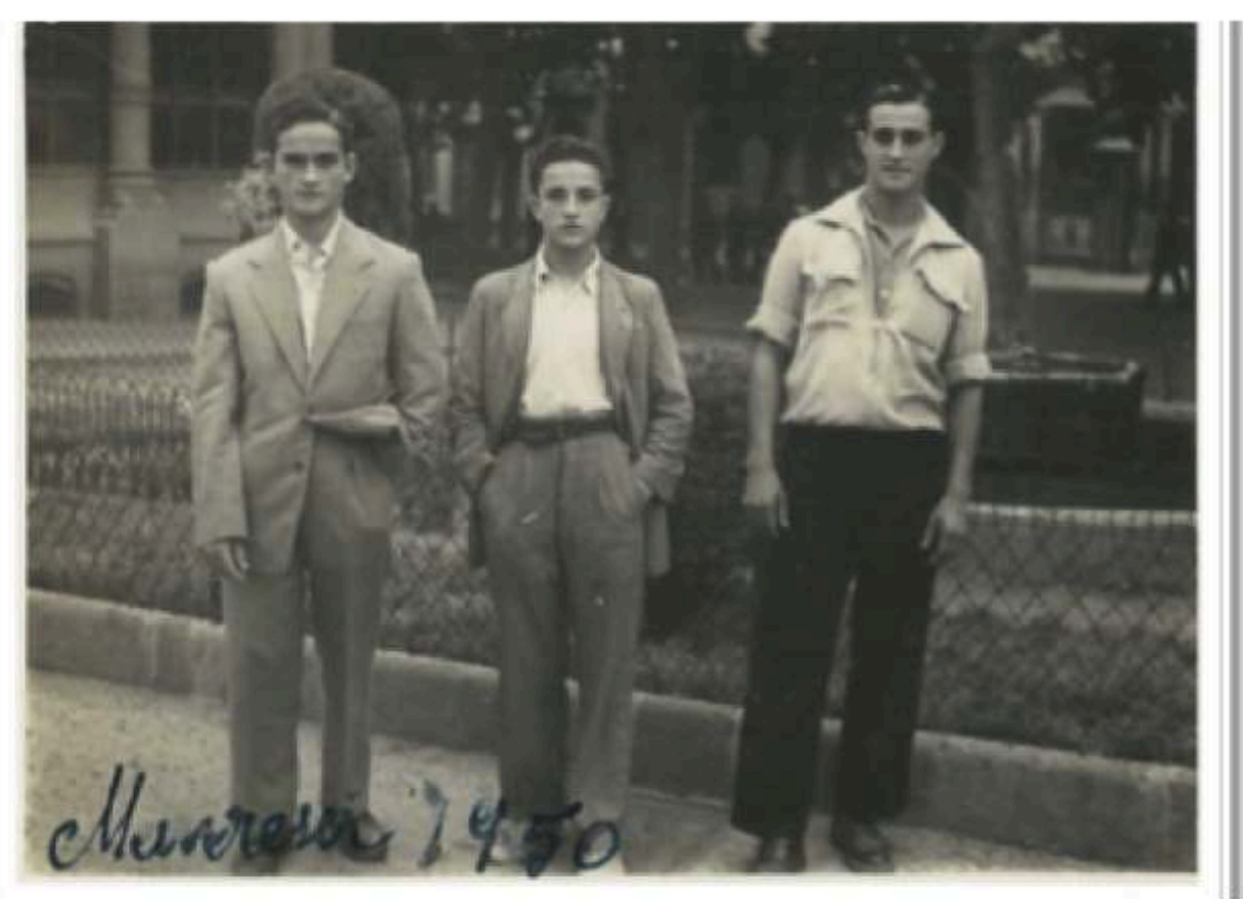

Source : Collection privée.

Julia et José se marient en 1954 et José bénéficiera à son tour du réseau de solidarité familial de la famille R.P. : Julia l'y encourage, il entrera à la FECSA.

Tous les membres de la fratrie de Julia s'établissent en Catalogne ; tous s'y marient et auront des enfants, recréant autant de noyaux familiaux. Comme pour la fratrie de José que nous avons moins approchée, on observe que la structure familiale se modifie dans la migration et s'adapte aux exigences de la nouvelle vie urbaine et active : tous passent du modèle de la famille élargie rurale à celui de la famille nucléaire, ayant tous un ou deux enfants. Pour la famille galicienne de Julia, Fruxil reste un point d'ancrage fort dans cette phase de son parcours. Elle y envoie son fils en 1958 au moment de la naissance de son deuxième enfant, Amélie, puis elle les laissera tous les deux au moment de partir pour la France. La disparition accidentelle en 1974 du frère ainé, José R.P., l'héritier resté auprès des parents en Galice, transférera définitivement l'ensemble de la famille d'origine à Manresa, à l'exception de Julia qui a émigré en France en 1962.

\section{Deuxième étape : de Manresa à Reims, pionnière en France}

Il semble que des démêlés judiciaires de son mari qui restent à élucider, aient contribué à la décision de Julia d'émigrer à nouveau, en plus de conditions de vie qu'elle juge défavorables : en tant que femme mariée elle n'a pas un accès libre à l'emploi ${ }^{16}$ et sa situation familiale est très instable. L'émigration vers l'étranger avait repris avec la signature d'accords bilatéraux entre l'Espagne et certains pays européens dont la France à partir de 1948 et la création de l'Institut de l'émigration (IEE) en 1956 montre que le régime a besoin de contrôler les flux migratoires très massifs. C'est une opportunité, Julia y voit de nouvelles perspectives pour aller de l'avant, caractéristique 
qu'Amélie, sa fille, décrit comme l'un des traits dominants de sa personnalité. Sa première employeuse avec qui Julia est restée en contact après son mariage lui indique l'adresse d'une famille française à la recherche d'une employée de maison. Les « bonnes » espagnoles ont bonne réputation en France dans les années 1960 (Tur, 2003, p. 95). Julia part, seule, avec une valise, une adresse en poche et un petit dictionnaire que ses enfants conservent comme « une trace de l'histoire de [leur] mère ».

Julia les laisse à la charge de sa famille galicienne « le temps de m'installer, dit-elle, de trouver un endroit où vivre ", un logement pouvant accueillir la famille dont le premier employeur ne veut pas $^{17}$. Évoquant ce moment marquant où pour la première fois elle est séparée de sa mère - elle mentionne très peu son père qui semble absent du quotidien -, Amélie dit: "c'est le premier déracinement, nous sommes éloignés de notre univers familier, Manresa, où nous sommes nés et où nous avons commencé à avoir notre vie ». Mais le choc de la première séparation semble avoir été dépassé par l'expérience du séjour galicien : elle et son frère découvrent un monde, ils apprennent, en plus des deux langues qu'ils parlaient déjà, le catalan et le castillan, le galicien et deviennent trilingues ${ }^{18}$; au village, il n'y a pas l'eau courante et l'électricité ne sera installée qu'après leur départ, mais les bons souvenirs s'accumulent. Amélie et son frère évoquent ce séjour comme une véritable émigration /immigration : nés dans une ville industrielle en Catalogne ils se retrouvent dans un village rural de la Galice profonde, dont leur mère est originaire, et découvrent des modes de vie, une langue et une culture très éloignés de ce qui faisait leur quotidien en ville. Amélie confie la rapidité de l'adaptation qui les transforme en «petits Galiciens»: tous les enfants participent aux travaux des champs, ils mènent les vaches aux prés, avec leurs cousins, passent des journées au grand air ; les animaux de la ferme leur deviennent familiers; ils ne vont pas ou peu à l'école, seulement quand les travaux des champs le permettent, comme les enfants du village ; ils sont entourés et vivent en famille malgré l'absence de leurs parents. Ils découvrent une vie liée aux rythmes des travaux des champs et à la terre, des habitus sociaux ruraux dans lesquels Amélie voit des "savoirs ancestraux ", des nourritures... ils s'intègrent. Même si l'absence de leur mère reste «inexpliquée et inquiétante " pour elle, ils apprécient les logiques de la famille élargie aux trois générations qui cohabitent et la présence nouvelle d'adultes nombreux auprès d'eux : «il y avait toujours quelqu'un avec nous, toujours quelque chose à faire ${ }^{19}$. Elle souligne de manière récurrente la dimension de la découverte de modes relationnels différents. C'est pour cette raison aussi qu'elle rapporte avoir vécu le départ vers la France comme une nouvelle rupture avec un monde au sein duquel elle et son frère ont fait leur première expérience d'intégration réussie.

Le séjour a duré onze mois. Leur oncle José les mène à dos d'âne rejoindre l'autobus où leur mère les attend; il les rassure et dessine avec ses mots un avenir prometteur. Ils vont faire le long voyage en train qui les conduira en Champagne, au Château de Vrilly. 
Figure $n^{\circ} 9$ : Le Château de Vrilly, carte postale ancienne

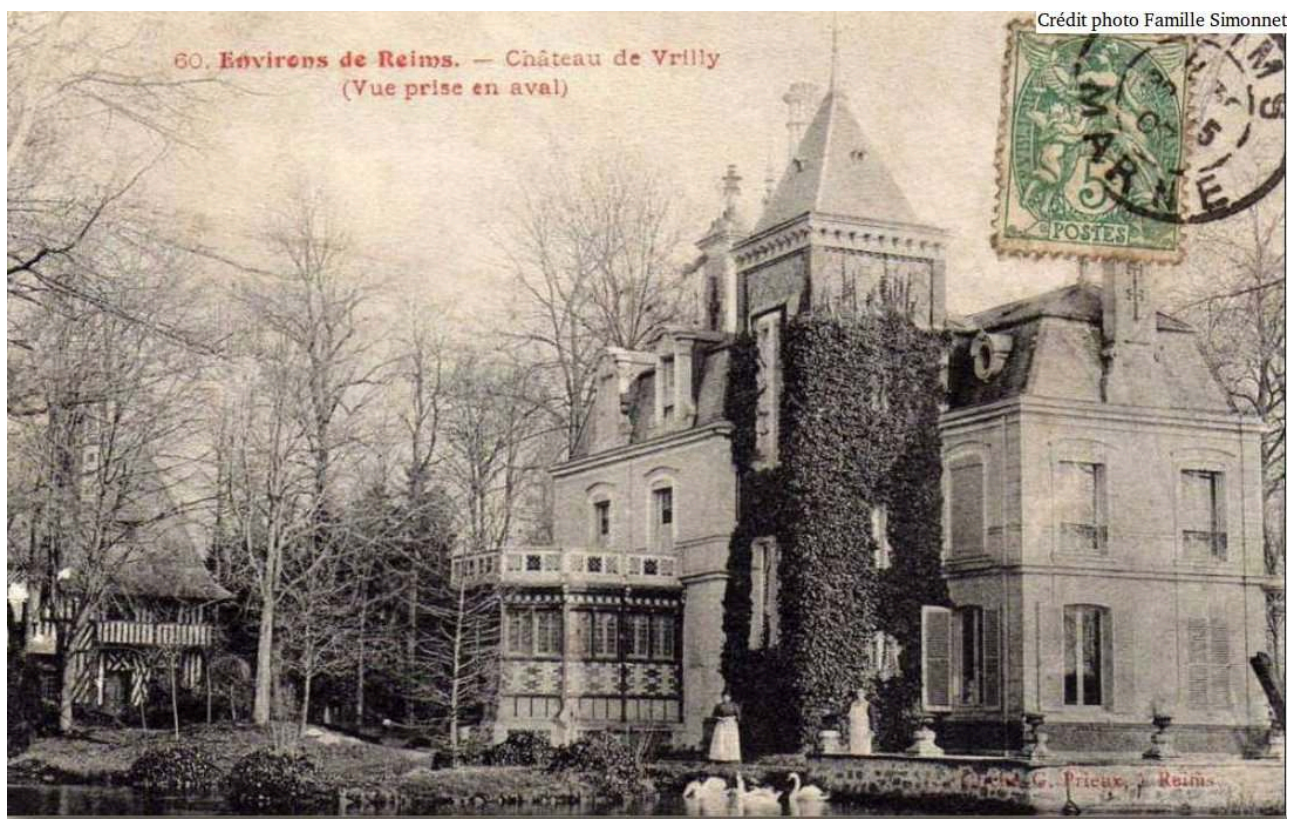

Source : Collection privée.

30 Nouveau pays, nouvelle langue, nouvelles habitudes... la rupture est brutale pour Amélie qui, à six ans, suit les périples de sa mère, qui avait, elle, souhaité quitter son village à 16 ans. La nouvelle vie a des avantages : l'eau courante, l'électricité et l'école française. Mais le quotidien d'Amélie est bouleversé :

Le changement a été brutal. Je ne comprenais pas la langue... En pension j'étais punie sans comprendre pourquoi, la nourriture était ... si différente ...

Les conditions économiques de la famille sont difficiles et les employeurs n'acceptent pas les enfants... Ils sont d'abord placés chez une nourrice, puis dans une «École de plein-Air », un pensionnat ; Amélie fait « l'expérience la plus traumatisante de [sa] vie » dans un collectif dont elle ignore les codes. Loin de ses cousins galiciens et catalans, qu'elle ne reverra qu'après plusieurs années, séparée de son frère qui est plus grand, elle vit « une sorte d'exil intérieur » marqué par la solitude et la distension de tous les liens familiaux. Les contours de son environnement familial se réduisent jusqu'à disparaître avec son entrée au pensionnat. Des mois plus tard, lorsque leurs parents auront trouvé un logement pour tous, elle retrouvera un milieu familial : Nous dormions tous dans la même pièce et le matin, nous devions plier fauteuils et canapé. C'était le salon! Des souvenirs positifs commencent à lui revenir en mémoire : C'est là que nous avons eu la télé!

31 En 1971, la famille déménagera à Toulouse puis dans la banlieue toulousaine où ils achèteront un pavillon et se fixeront définitivement... avant le retour de Julia et José en Espagne en $1978^{20}$ et leur divorce. Amélie et son frère vivront une adolescence toulousaine où ils se voient comme "des immigrés intégrés", presque plus des immigrés : ils ont les difficultés de tous les adolescents, ne vont plus en Espagne que pendant l'été, puis plus du tout... ils oublient leurs origines. Jeunes adultes ils tenteront de se rapprocher à nouveau de leur famille espagnole, mais ils n'ont plus rien de commun avec leurs cousins, leurs oncles et tantes, leurs grands-parents qu'ils ne 
reverront qu'une fois avant leur décès. Leurs passages sur la route des vacances, permettent de renouer des liens affectifs épisodiques et ténus, des moments pleins d'émotions mais qui s'évanouissent avec le retour dans leur vie française. Ils deviennent des Français. Amélie acquiert la nationalité à 18 ans par «naturalisation ». La rupture est définitive avec la plupart des membres de leur famille espagnole. Ils ne seront jamais tout à fait d'ici ni vraiment de là-bas ; bien plutôt des deux à la fois... Ce sont pourtant ces liens très ténus qui caractérisent la manière particulière dans laquelle s'établit une forme de «famille dans la distance » (Imbert et al., 2018).

Cette deuxième étape de l'émigration familiale repose sur des contraintes que la mère semble avoir assumées seule. Les conditions d'une «vie meilleure» en France dépendront d'elle: emplois, logement, scolarisation des enfants dans un système scolaire français "indéniablement accueillant $»^{21}$ selon Amélie et son frère. Si généralement la migration de Julia et de sa famille est considérée comme réussie sur le plan économique relativement à sa condition d'origine, le témoignage d'Amélie et son bilan négatif sur le plan des liens affectifs familiaux qu'elle présente comme "brisés » et perdus pour elle, nous amène à relativiser humainement cette réussite. Amélie relate un parcours fait de ruptures et de recommencements qui révèle ses difficultés dans un processus d'adaptation à répétition.

\section{Des gains et des pertes, un bilan familial contrasté}

Dans le cas de Julia, pionnière de sa génération, on peut affirmer que l'émigration a permis des gains sociaux substantiels en termes de niveau de vie et d'acquis culturels : Julia s'est insérée par le travail, a accédé à la propriété puis à la multipropriété, en Espagne et en France, et ses enfants ont fait des études supérieures ${ }^{22}$. Mais sur le plan humain, Julia a vécu en déracinée, elle a peu fréquenté ses sœurs restées en Catalogne et elle a fini par divorcer. Ses enfants ont vécu loin d'elle après qu'elle est retournée en Catalogne à la faveur d'une politique d'aide au retour en 1978. Le lien familial s'est distendu et a provoqué un accroissement de l'isolement des acteurs. Un fossé culturel s'est creusé entre elle et ses enfants, sans doute pire encore avec leur père. Pourtant, à l'heure de la retraite, elle est retournée dans son village, "al pueblo », et y a retrouvé de l'aisance et un certain bonheur, retrouvant les mêmes voisins, certaines habitudes sociales, avec le confort en plus, contribuant d'ailleurs à le faire arriver jusqu'au village ${ }^{23}$. 
Figure $\mathrm{n}^{\circ} 10$ : Julia, retour à Fruxil vers 2003

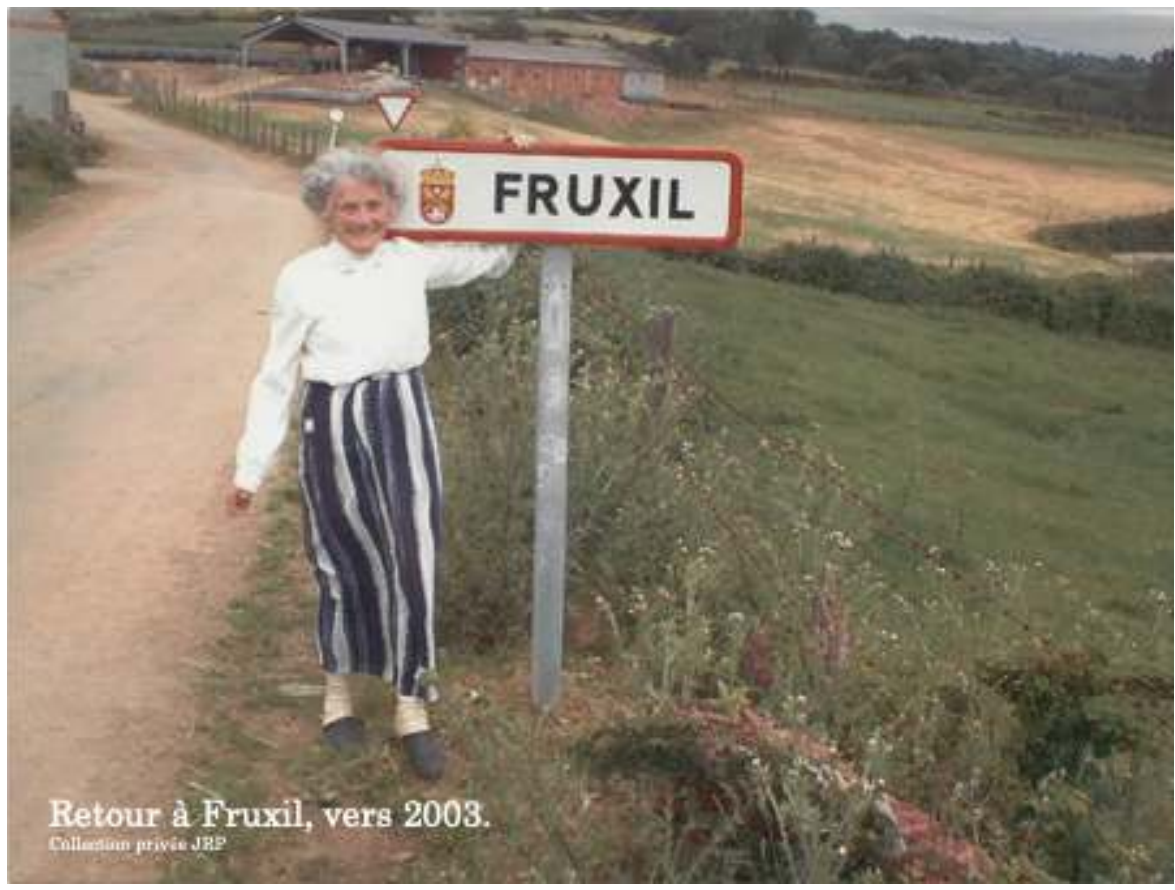

Source : Collection privée.

Figure $\mathrm{n}^{\circ} 11$ : Les bâtisses actuelles de la famille R.P., Fruxil, vers 2003

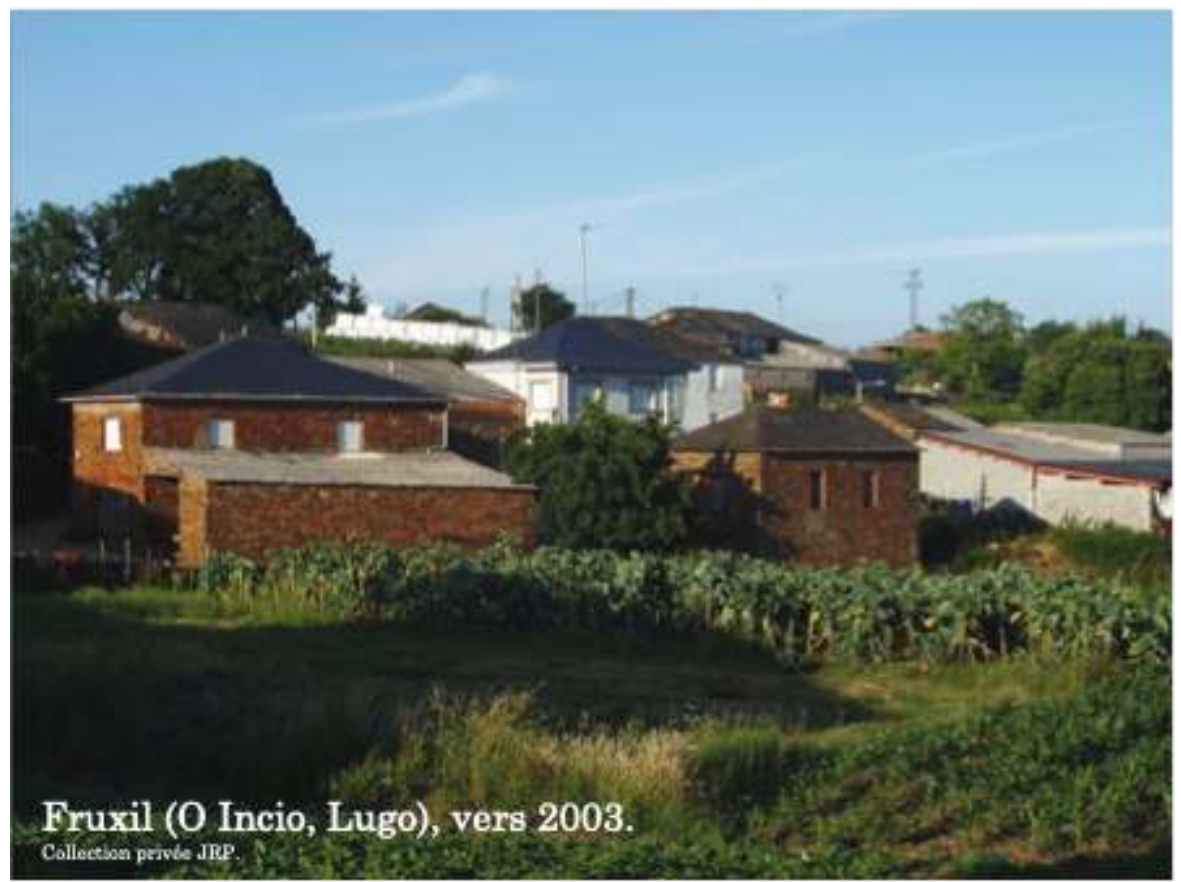

Source : Collection privée.

34 Julia dit y avoir passé les meilleures années de [sa] vie. En effet, à l'heure du grand âge et de la dépendance, elle fait dans une certaine indifférence, l'expérience migratoire de trop : comme l'a montré Abdelmalek Sayad pour l'immigration algérienne, la présence/ absence séquentielles, ici et là, ne lui permettent plus de se sentir ni d'ici ni de là-bas 
(Sayad, 2006). Son dernier « retour» est celui où les souvenirs s'estompent cependant que des liens familiaux se renouent : elle vit plus près de ses petits-enfants comme de ses enfants; ils n'ont plus de lien avec la terre d'où elle est partie, la Galice, mais la famille joue son rôle de soutien du grand âge. Des études récentes, notamment statistiques, mettent au jour et documentent l'évidence du processus et « la banalité de l'existence des familles transnationales ", ce que notre exemple et son évolution dans le temps sont venus étayer. Il reste à interroger la durabilité et la variabilité des processus, car «le transnationalisme familial ne se transmet pas ou très peu » (Lacroix ; Audebert ; Ma Mung, 2018).

35 Qu'en est-il de l'évolution des régions d'origine et d'accueil de la migration? L'exploitation des sols et des bêtes utilise les mêmes techniques et les méthodes modernes qu'ailleurs, la question de la transition écologique s'y pose comme ailleurs. Les usages et les traditions populaires de la société rurale de la Galice profonde sont conservées dans un Musée, $O$ Museo do povo galego (Musée du peuple galicien ${ }^{24}$ à Saint-Jacques de Compostelle, où des objets et des outils dont les jeunes immigrés des années 1960 ont connu ou vu l'usage sont exposés; certaines traditions sont classées au patrimoine immatériel de l'humanité comme celle des Danzantes, les danseurs, une tradition préservée et maintenue vivante dans la commune par des jeunes gens qui y ont une forte implication (Rodriguez Aira, 2011).

Figure n 12 : Danzantes, lors de la "fiesta mayor", la fête du village, 2016

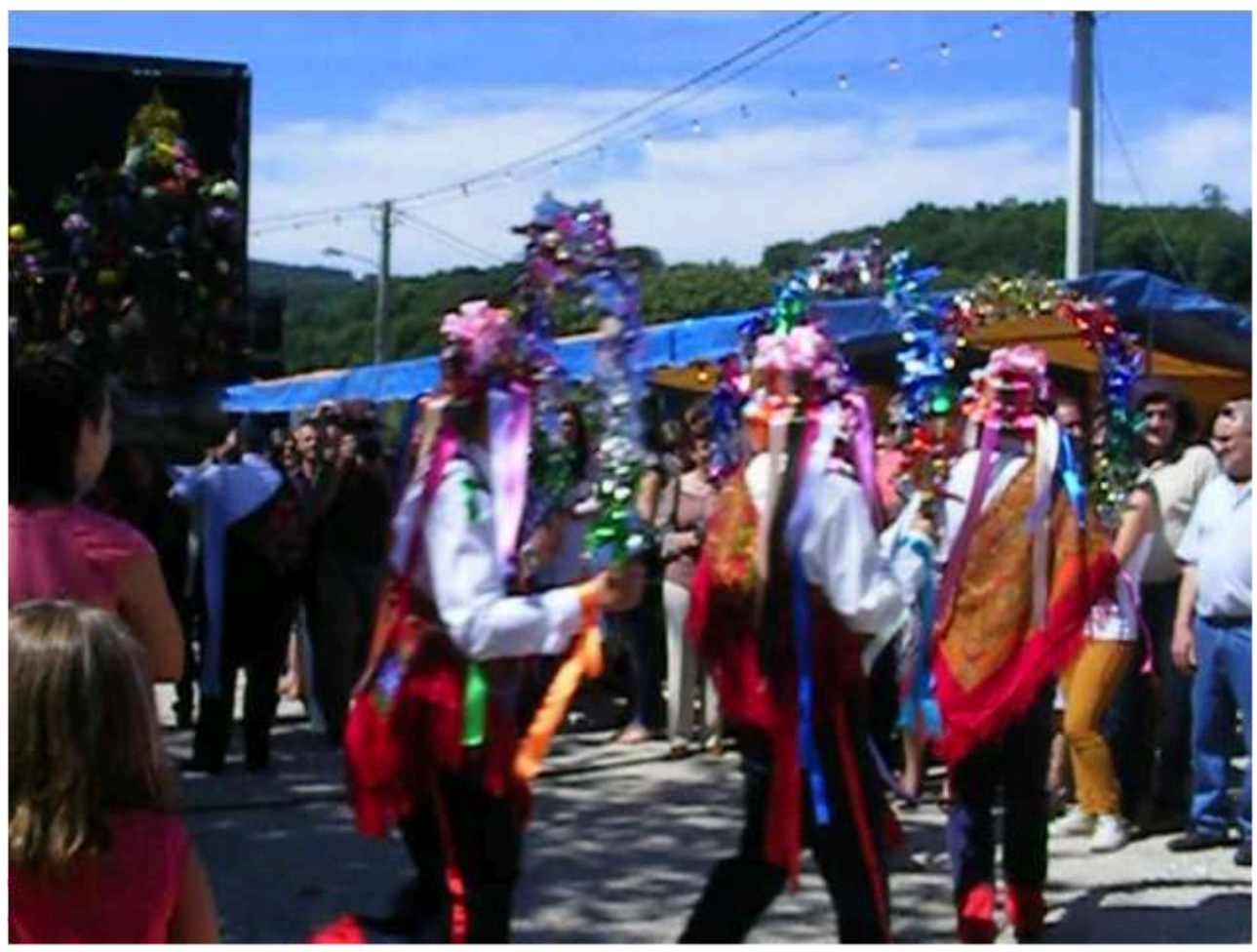

Source : Collection privée.

Quant à la Catalogne industrielle, elle a vu ses grandes usines du XIXe siècle abattues, parfois heureusement réhabilitées comme le bâtiment moderniste des anciens abattoirs de Manresa en une bibliothèque universitaire singulière. L'imposant patrimoine industriel plus ou moins valorisé à ce jour trouve progressivement de possibles 
réhabilitations et son immobilité de pierre témoigne tant des effets des crises économiques que de la mutation des sociétés industrielles.

Figure $n^{\circ} 13$ : Manresa, la Fábrica Nova, destruction.

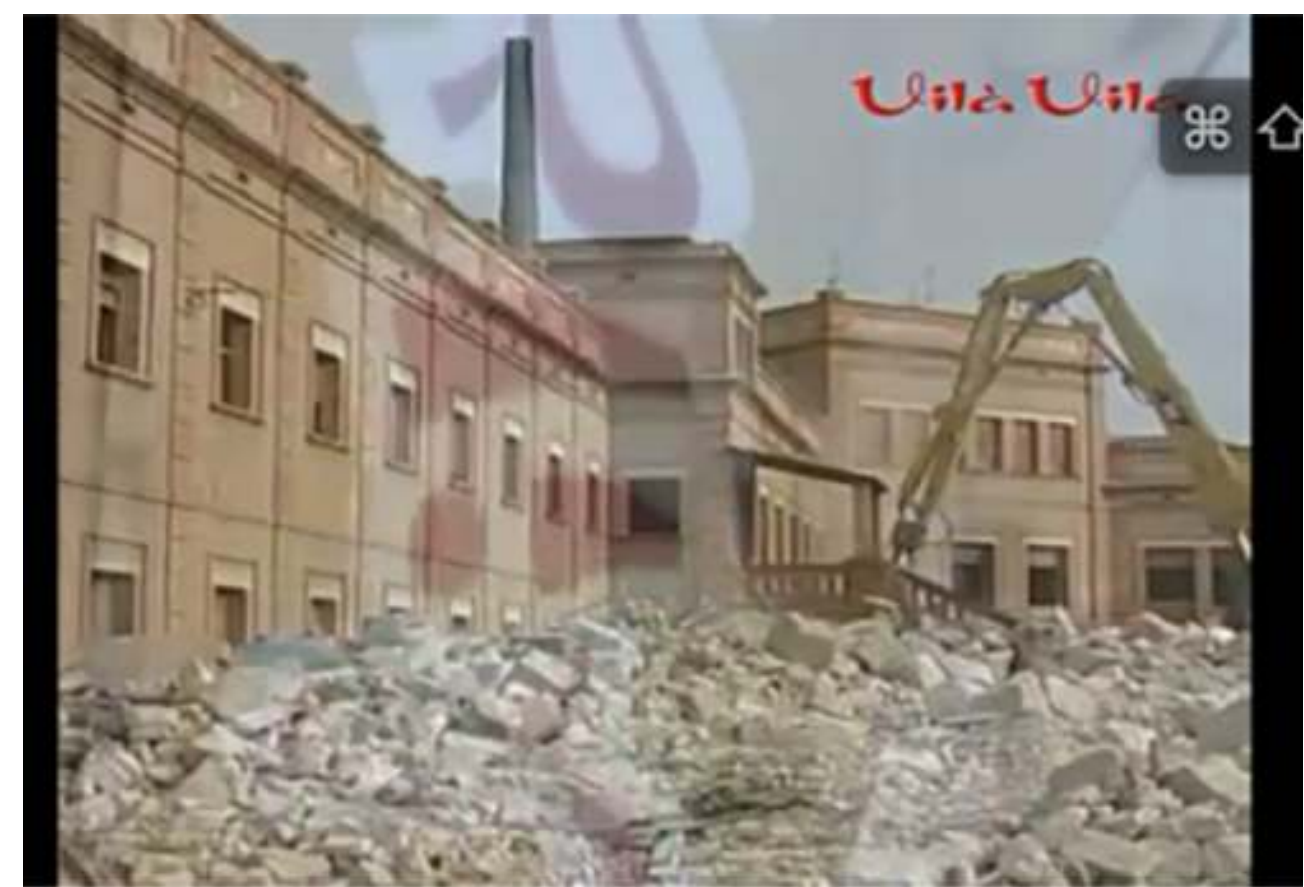

Photogramme du reportage Derribo Fábrica Nova de Manresa, réalisé par Vila Vila Enderrocs, $2013^{25}$.

Deux mondes qui naguère vivaient sur des rythmes économiques différents et complémentaires sont tous deux entrés dans la mondialisation et ont suivi comme ailleurs le rythme d'une modernisation à marche forcée. Cependant, les techniques ont agrandi les exploitations, ceux qui sont restés ont prospéré, notamment grâce aux transferts monétaires des émigrants, les «remises» ("remesas») de celles et ceux qui sont partis mais qui ont alimenté l'économie locale par leurs apports financiers. Cet intérêt matériel de l'émigration, que Franco comprit dans la deuxième période de la dictature ${ }^{26}$, est un élément important de l'histoire migratoire de l'Espagne et de la Galice. Mais les émigrants ont aussi contribué à la modernisation des mentalités, par leurs retours pendulaires ou définitifs (Fernandez Vicente, 2004). Les maisons qu'ils ont bâties au retour, où ils ont pris leur retraite parfois, sont plus grandes, ils ont réinvesti leurs gains dans le pays et leurs enfants, souvent les femmes aujourd'hui, agrandissent les exploitations. 
Figure $n^{\circ} 14$ : Manresa, La Bibliothèque universitaire, réhabilitation du bâtiment moderniste des abattoirs municipaux

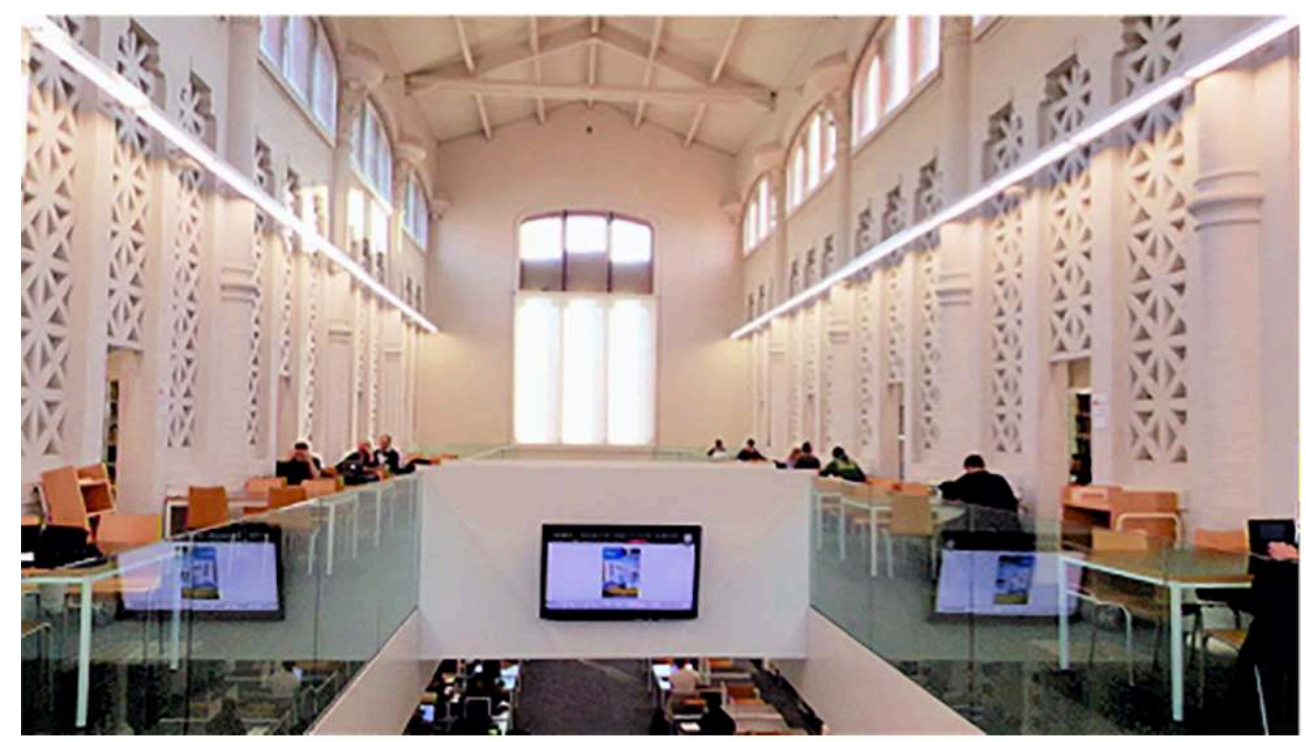

Source : Collection privée, 2016.

De ce qu'Amélie nommait "des savoirs ancestraux », les générations actuelles ne savent que peu de choses: les vidéos publiées sur internet par des associations qui travaillent, là-bas, à préserver et à faire connaître un patrimoine historique ou culturel galicien, un musée. Depuis l'étranger, les jeunes les regardent comme des manifestations plus ou moins folkloriques d'un monde patrimonialisé. Au mieux, ils cherchent à maintenir une part de la mémoire d'un monde dont ils ne font pas partie mais ils n'ont plus aucune contribution à son devenir si ce n'est, par leur vote quand, comme Amélie, ils ont récupéré la double nationalité. Les jeunes ont fait des études et sont devenus des exploitants contrairement à leurs grands-parents qui étaient des "labradores", les "laboureurs", les paysans d'antan. Les différences sociales sont globalement conservées dans le village de Julia où, globalement, tout le monde vit mieux.

Le bilan des pertes, des gains et des transformations de la migration de Julia observés au prisme de la famille, entre la vision sombre d'Amélie et l'observation qu'objectivement nous faisons de liens familiaux transformés mais maintenus dans une forme de "famille à distance", oblige sans négliger l'expérience individuelle, au constat de bénéfices indéniables : le statut social de Julia n'a cessé d'évoluer, donc celui de ses enfants. La structure et la nature des liens familiaux ont changé, mais une famille reste constituée, qui à chaque étape, s'est adaptée. Bien que marqués par le fait migratoire qui continue de se raconter et de se chercher un sens, les individus ont tous accédé à des positions plus élevées et plus libres que ne l'aurait permis la société galicienne au point de départ de la migration, à Fruxil, en 1940.

\section{Conclusion}

Nous avons choisi cette famille à l'occasion d'une exploration de terrain pour son parcours complexe, depuis la fin des années 1930, et que nous avons pu suivre jusqu'en 2016 ; elle présente un profil migratoire assez classique dans l'histoire des migrations 
espagnoles et singulièrement galiciennes; en tant que tel, ce parcours reflète celui de nombreuses autres familles, dont les enfants, comme Amélie, ont fait l'expérience du déracinement. Par ailleurs, il reflète la profonde transformation de la structure familiale, à l'occasion de la migration et en s'adaptant aux contraintes du monde moderne, passée du modèle de la famille "élargie", où plusieurs générations cohabitent, au modèle de la famille "nucléaire ", où seuls parents et enfants vivent ensemble.

41 À partir de l'exemple de cette famille, nous avons montré que l'émigration reste une épreuve de rupture, une dystopie confrontée à l'utopie des représentations qui nourrissent les imaginaires des candidats à l'émigration des années 1940-1960. Cependant, à travers les mouvements pendulaires de ses membres émigrés, la famille a fait groupe y compris dans la distance, géographique ou temporelle. Des bénéfices économiques et culturels sont venus compenser des pertes de liens sociaux et familiaux. Nous avons ainsi distingué deux points de vue, celui de l'individu : Julia, José ou Amélie pour qui le bilan affectif contraste avec celui de la réussite matérielle de la famille. L'émigration ou l'exil provoque un sentiment profond et communicatif : la morriña des Galiciens dit cette mélancolie particulière que Rosalía de Castro excelle à chanter. Elle évoque ce qu'il faut de force aux émigrants - et aussi à ceux qui restent pour dépasser le sentiment de l'arrachement (de Castro, 1986). Même si aujourd'hui la globalisation des migrations a modifié les imaginaires, ce sentiment de perte est partagé par les migrants en situation de pauvreté, dans l'éloignement forcé et la séparation.

Enfin nous avons vu à travers cet exemple les catégories de la solidarité et de l'entraide familiale se jouer et montrer comment la famille tient et se tient dans la migration; garante d'une part d'humanité singulière, la famille se réinvente, sur place, à distance, constituant des familles qui sont de véritables «constructions sociales transnationales " (Faist, 2000 ; Gil Araujo, 2010). Dans ce même temps de la migration où les familles sont confrontées à des pressions contradictoires et aux contraintes de l'adaptation, les individus voient leurs origines leur devenir étrangères. Pour retrouver ces origines, les descendants tentent de renouer des fils de l'histoire. À contretemps des sentiments d'Amélie, nous avons montré comment une familialité humaine universelle entendue comme le lien primordial entre des individus d'une famille qui les pousse à continuer de « faire famille » dans la migration, ne semble pas être détruite. Cependant l'exemple montre également, même moins dramatiquement que pour les enfants de la guerre, comment la migration en suspend la réalisation. Les latences ou les intermittences dans ce parcours particulier viennent confirmer la perspective ouverte par la notion de "famille à distance" dans laquelle nous voulons voir, pour Amélie comme pour tous les enfants emportés dans les incertitudes de l'émigration / immigration familiale, une forme de réparation possible. 
Figure $n^{\circ} 17$ : Généalogie de la famille R.P.

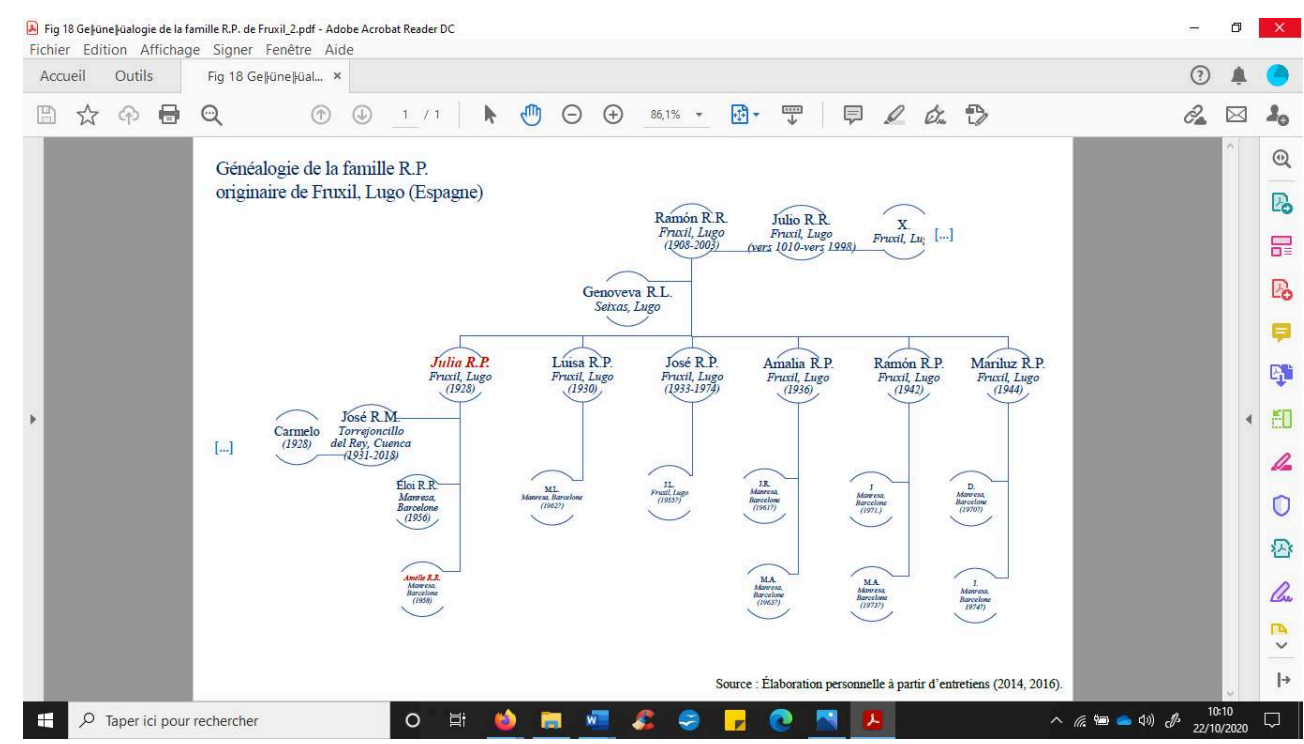

Source : Élaboration par l'auteure à partir d'entretien, 2014-2016.

\section{BIBLIOGRAPHIE}

Barciela López, Carlos (éd.) (2003) Autarquía y mercado negro : el fracaso económico del primer franquismo, 1939-1959, Barcelona, Crítica, 325 p. (Crítica/Historia del mundo moderno).

Barthes, Roland (1980) La chambre claire. Note sur la photographie, Paris, Éditions de l'Étoile ; Gallimard ; Le Seuil, 192 p.

Braudel, Fernand (1985) La dynamique du capitalisme, Paris, Arthaud, 120 p.

Canal, Jordi (dir.) et al. (2017) Histoire de l'Espagne contemporaine de 1808 à nos jours : politique et société, Paris, Armand Colin, 340 p. (U-Histoire).

Castro, Rosalía de (1986) ¡Prá Á Habana !, Follas novas V, Vigo, Galaxia, 205 p.

Cervantes López, Ginés ; Ramos Ramírez, Antonio (2016) Mémoires en exil, Lorient, Université Bretagne-Sud Lorient, [Site Web] réf. du 27 décembre 2018.

Chaput, Marie-Claude ; Llecha Llop, Canela ; Martinez-Maler, Odette (dir.) (2017) Escrituras de la resistencia armada al franquismo, Nanterre, Presses universitaires de Paris Nanterre, $350 \mathrm{p}$.

(Regards sur l'Espagne contemporaine).

Delfour, Christine (2015) L'Espagne, les autonomies et l'Europe, Essai sur l'invention de nouveaux modes d'organisation territoriale et de gouvernance, Bruxelles, Bruylant, 365 p. (Droit de l'Union européenne).

Dreyfus Armand, Geneviève (2015) Réfugiés espagnols, quand la France choisissait l'infamie, Libération, 9 septembre 2015, réf. du 7 janvier 2019 [Disponible sur Internet]. 
Dreyfus-Armand, Geneviève (1996) L'accueil des républicains espagnols en France : entre exclusion et utilisation ; 1936-1940, Matériaux pour l'histoire de notre temps, vol. 44, nº 1, pp. 36-41, réf. du 19 octobre 2020 [Disponible sur Internet].

Faist, Thomas (2000) The Volume and Dynamics of International Migration and Transnational Social Spaces, New-York, Oxford University Press, $112 \mathrm{p}$.

Fernandez Vicente, Maria José (2004) Émigrer sous Franco, Politiques publiques et stratégies individuelles dans l'émigration espagnole vers l'Argentine et vers la France (1945-1965), Paris, Université Paris 7- Denis Diderot, $494 \mathrm{p}$.

Th. Doct. : Hist. : Paris : 2004.

Ferrol, Manuel, Photographer, Site officiel, réf. du 12 mai 2020 [Disponible sur Internet].

Ferrol, Patricia (2016) A memoria gráfica da emigración española, [commentaire sur les photographies de l'exposition, 18 février - 9 avril 2016] Fundación Galicia Obra Social [Disponible sur Internet].

Formiguera, Pere ; Cánovas, Carlos (photographe) (1992) Le Temps du silence : la photographie espagnole des années 50 et 60, Barcelone, Fundació Caixa de Catalunya, 255 p. (Catalogue de l'exposition du 21 avril au 31 mai 1992, Centre d'Art Santa Mònica, Barcelone ; du 17 novembre 1992 au 1er février 1993, Palais de Tokyo, Paris).

García Barbancho, Alfonso (1967) Las migraciones interiores españolas en 1961-1970, Madrid, Ministerio de la Presidencia, Secretaria General Técnica, 216 p.

García Barbancho, Alfonso (1979) Disparidades regionales y ordenación del territorio, Barcelone, Ariel, $272 \mathrm{p}$.

Gil Araújo, Sandra (2010) Políticas migratorias, género y vida familiar. Un estudio exploratorio del caso español, in Grupo Interdisciplinario de Investigador@s Migrantes (coord.) Familias, niños, niñas y jóvenes migrantes. Rompiendo estereotipos, Madrid, IEPALA, pp. 81-92.

Hochstadt, Steve (1999) Mobility and modernity: migration in Germany, 1820-1989, Ann Arbor, University of Michigan Press, 331 p. (Social history, popular culture, and politics in Germany).

Imbert, Christophe; Lelièvre, Eva; Lessault, David (dir.) (2018) La famille à distance. Mobilités, territoires et liens familiaux, Paris, INED, 376 p. (Questions de populations, 2).

INSEE (2012) Fiches thématiques : Éducation et maitrise de la langue, Immigrés et descendants d'immigrés en France, Paris, INSEE Références, pp. 160-177, réf. du 2 juin 2020 [Disponible sur Internet].

Lacroix, Thomas ; Audebert, Cédric ; Ma Mung, Emmanuel (2018) La famille transnationale dans les recherches sur les migrations, In Imbert, C.; Lelièvre, E.; Lessault, D.(dir.) La famille à distance. Mobilités, territoires et liens familiaux, Paris, INED, pp. 73-92 (Questions de populations, 2).

López-Pozos, Cecilia (2009) El costo emocional de la separación en niños migrantes : un estudio de caso de migración familiar entre Tlaxcala y California, Agricultura, sociedad y desarrollo, vol. 6, pp. 81-103, réf. du 13 mai 2020 [Disponible sur Internet].

Marín-Dòmine, Marta (2013) La transmission et la construction de l'espace transgénérationnel, Témoigner. Entre histoire et mémoire, $\mathrm{n}^{\circ}$ 115, pp. 97-107, réf. du 7 décembre 2019 [Disponible sur Internet]

Martínez López, Francisco « El Quico » (2000) Guerrillero contra Franco. La guerrilla antifranquista del León (1936-1951), Paris, Syllepse, 175 p. 
Moradiellos, Enrique (2012) La guerra de España (1936-1939) : estudios y controversias, Barcelona, RBA Libros, 379 p. (Temas de actualidad. Historia de España).

Murado, Miguel-Anxo (2008) Otra idea de Galicia, Barcelona, Debate, 207 p.

Navarro, Vicenç (2008) Los niños perdidos del franquismo, El País, 24 décembre 2008, réf. du 16 novembre 2019 [Disponible sur Internet]

Noiriel, Gérard (1988) Le creuset français. Histoire de l'immigration XIXe-XXe siècle, Paris, Éditions du Seuil, 437 p. (Points).

Núñez Seixas, Xosé Manoel (2005) Otras miradas a la historia de la emigración gallega : sobre cartas, memorias y fotos, Estudios Migratorios Latinoamericanos, vol. 19, $\mathrm{n}^{\circ}$ 58, pp. 483-504.

Núñez Seixas, Xosé Manoel (2016) O soño da Galiza ideal : estudos sobre exiliados e emigrantes galegos, Vigo, Galaxia, 276 p.

Oris, Michel (2003) The history of migration as a chapter in the History of the European Rural Family: An Overview, The history of the family, vol. 8, n², pp. 187-215,

Oso Casas, Laura (2004) Españolas en Paris, Estrategias de ahorro y consumo en las migraciones internacionales, Barcelona, Edicións Bellaterra, 266 p.

Porrúa, María del Carmen (2012) El tema de la emigración en la poesía de Rosalía de Castro y su proyección en dos poetas gallegos, Actas do Congreso Internacional de estudios sobre Rosalía de Castro e o seu tempo (III), Santiago de Compostela, Consello da Cultura Galega ; Universidade de Santiago de Compostela, réf. du 27 décembre 2018 [Disponible sur Internet].

Prost, Antoine ; Cytermann, Jean-Richard (2010) Une histoire en chiffres de l'enseignement supérieur en France, Le Mouvement Social n² 233, pp. 31-46.

Rodriguez, José Julio ; Ribas, Adolfo (2009) Memoria gráfica de la emigración española, Madrid, Dirección general de la Ciudadanía Española en el Exterior, 162 p. (Catalogue de l'exposition éponyme), consulté le 27 décembre 2018 [Disponible sur Internet].

Rodriguez Aira, Miguel Angel (2011) Danza Da Cervela Parte 1 et Parte II.avi, Fundación TIC Lugo, Reportage en deux parties, réf. du 6 janvier 2019 [Disponible sur Internet : Partie I et Partie II].

Rubio-Milet, Emma (en cours) Galiciennes en Catalogne. Migrations économiques inter-régionales pendant le premier franquisme (1939-1964), Paris, Université Gustave Eiffel (ex-UPEM), Th. de Doct. : Etud. Hisp. : en cours.

Rubio, Javier (1977) La emigración de la guerra civil de 1936-1939: historia del éxodo que se produce con el fin de la II República española, Madrid, Librería Editorial San Martín, 3 vol. , 1229 p.

Sayad, Abdelmalek (2006) L'immigration ou les paradoxes de l'altérité, 2. Les enfants illégitimes, Paris, Raisons d'agir, 216 p. (Cours et Travaux).

Sierra-Paycha, Célio (2018) La famille à distance, ressource spatiale pour migrer et support de flux transnationaux, In Imbert, C. ; Lelièvre, E. ; Lessault, D. (dir.) La famille à distance. Mobilités, territoires et liens familiaux, Paris, INED, pp. 93-114 (Questions de populations, 2).

Tur, Bruno (2003) De Valence à Paris : Itinéraires des « bonnes » espagnoles (1940-1974), Paris, Université Paris VII, $199 \mathrm{p}$.

Mém. Maîtr. : Hist. Géogr. : Paris : 2003.

Tur, Bruno (2014) L'immigration espagnole à Paris dans les années 1960 : discours, représentations et stéréotypes, Nanterre, Université Paris-Ouest Nanterre La Défense, 637 p., Th. Doct. : Lettr. Lang. Spect. : Nanterre : 2014. 
Vallon, Serge (2006) Qu'est-ce qu'une famille ? Fonctions et représentations familiales, Vie sociale et traitements (VST), $\mathrm{n}^{\circ} 89$, pp. 154-161 [Disponible sur Internet].

Yusta Rodrigo, Mercedes (2017) La historiografía de la guerrilla antifranquista : Debates y combates, In Chaput, M.-C. ; Llecha Llop, C. ; Martinez-Maler, O. (dir.) Escrituras de la resistencia armada al franquismo, Nanterre, Presses universitaires de Paris Nanterre, 350 p. (Regards sur l'Espagne contemporaine).

Zola, Émile (1985) La fortune des Rougon, Paris, Librairie générale française, 414 p. (Le Livre de Poche).

\section{NOTES}

1.

L'expression « la drôle de guerre » correspond au début de la seconde guerre mondiale où aucune bataille n'a eu lieu pendant de longs mois entre la déclaration de guerre (septembre 1939) et l'invasion de la Belgique par les troupes nazies (mai 1940), les Alliés ayant assisté à la défaite de la Pologne sans bouger. Pour nous, la paix qui aurait dû s'installer en Galice est par analogie une «drôle de paix » et, en fait, l'imposition du régime dictatorial.

2.

Voir également sur le sujet: Armengou, Montse et Belis, Ricard (2006) Els nens perduts del franquisme, TV3, disponible sur Internet : <https://vimeo.com/search ?q =els \%20nens \%20perduts \%20del \%20franquisme>, consulté le 16 novembre 2019.

3.

Le terme "minyones» est employé en Catalogne pour désigner les employées de maison. Transcrit en espagnol par "miñonas", on le retrouve dans les usages et dans la littérature espagnole, tout comme dans les travaux de recherche universitaire. Voir, pour les années 1960 en France, les travaux sur les «bonnes" espagnoles, de Laura Oso Casas (2004) ou de Bruno Tur (2003 et 2014).

4.

«El tópico ha ocupado la poesía y la prosa, la ficción y el ensayo, desde diferentes ángulos y con distinta intensidad. Entre los poetas gallegos el tema de la emigración tiene muchos exponentes. » [nous traduisons].

5.

Native de Saint-Jacques de Compostelle, Rosalía de Castro (1837-1885) est l'auteure centrale du Rexurdimento, mouvement de « récupération de la langue et de la culture galiciennes au milieu du XIXe siècle.» (Diccionario da lingua Galega, https://academia.gal/dicionario/-/termo/ rexurdimento, consulté le 27 décembre 2018) ; adoptant le point de vue de celle qui est restée sur sa terre qu'elle ne quitte en effet que durant quelques années pour Madrid où l'activité littéraire appelle son mari Miguel Murguía, autre figure essentielle du Rexurdimento, elle produit une œuvre poétique et romanesque en galicien, enracinée dans sa galeguidade (« galicianité »). Dans le poème intitulé «¡Prá Á Habana!» de son recueil Follas novas, elle chante par exemple singulièrement l'émigration comme un fléau qui vide « la Galice de ses hommes /qui puissent la travailler ", de ses forces vives (de Castro, 1986).

6.

[...] « el reflejo de su propia situación personal y de la situación política, social y económica que lo empujo al desarraigo. » [nous traduisons].

7. 
Doctrine politique basée sur les principes d'un attachement à la Galice fondé sur la défense et l'amélioration des aspects politiques, sociaux et culturels qui constituent son identité nationale, (Diccionario da lingua Galega, https://academia.gal/dicionario/-/termo/busca/galeguismo, consulté le 27 décembre 2018).

8.

Alfonso Daniel Rodriguez Castelao, dit Castelao (1886-1950), écrivain et homme politique, il contribua avec un groupe d'intellectuels galiciens à la constitution et à la reconnaissance de l'identité, de la culture et de la langue galiciennes dans un statut rédigé et plébiscité en Galice à la veille de la guerre d'Espagne, et voté par le parlement de la République en juillet 1938.

9.

Le punctum est le concept développé par Roland Barthe dans La chambre claire pour tenter de saisir ce qui, dans un cliché, provoque l'émotion unique, l'adhésion.

10.

Le déroulé du parcours migratoire et les éléments de l'histoire de Julia sont tirés des entretiens menés en 2014 et en 2016 en Galice, en Catalogne et à Paris dans le cadre de notre thèse (en cours). Afin de respecter l'anonymat demandé par la plupart des personnes que nous avons rencontrées ou interviewées, tous les prénoms utilisés dans cet article à l'exception de celui de Julia et de ses parents ont été modifiés.

11.

D'autres familles du village racontent les histoires des grands oncles et tantes partis et définitivement restés en Argentine, à Cuba ou au Venezuela.

12.

La jeune sœur de Julia, interviewée en 2016 pour notre travail de terrain, nous a rapporté qu'il était réputé communiste. Nous n'avons vérifié cette réputation qu'auprès de certains membres de cette famille qui le confirment, mais des liens probables avec les milieux syndicaux d'avant la dictature nous semblent pouvoir justifier ses capacités à informer ses proches immigrés en Catalogne sur les postes de travail disponibles rapidement.

13.

La production électrique est l'une des activités de la ville qui continuera d'absorber des générations d'« immigrés » de l'intérieur comme la totalité des hommes de la famille R.P.

14.

Julia, entretien de 2014 : “ ¡yo quería marchar de Fruxil ! Debía tener 10 años cuando ya me quería ir, sí, quería dejar aquel barro, porque entonces no estaba asfaltado. Yo tenía una rabia a aquellos zocos que llevábamos y me quería marchar nada más por eso... » [nous traduisons].

15.

José est décédé en 2016, nous ne l'avons pas rencontré. Ces propos sont rapportés soit par son frère aîné qui vit en Catalogne et que, sur indication de Julia nous avons pu rencontrer à l'occasion d'un séjour de recherche, soit par l'intermédiaire d'Amélie, sa fille.

16.

La première des «Lois fondamentales » édictées par Franco est le « Fuero del trabajo » («For du travail »,1938), loi d'inspiration fasciste qui avait pour objectif d'encadrer les travailleurs capables de s'organiser. Elle dissout les syndicats et interdit le travail aux femmes mariées pour, selon la rhétorique de son inspiration, «libérer la femme mariée de l'atelier et de l'usine » (Canal, 2017, p. 210).

17.

Les employées de maison, comme en Espagne mais sans l'interdiction de travailler si elles sont mariées, sont idéalement célibataires et sans enfants. Voir sur ce thème le film Les femmes du bème étage, de Philippe Le Guay ou les travaux de Bruno Tur $(2003,2014)$.

18. 
Malgré la tentative infructueuse du régime de Franco d'interdire totalement les langues du pays (galicien, catalan, basque) qu'il dégrade en « régionales ", il ne put en faire disparaître l'usage dans les familles et les ateliers, mais elles furent minorisées.

19.

Tous les propos d'Amélie sur son expérience rapportés ici sont issus de notes de terrain et d'un entretien réalisés en 2014. De même, les éléments de l'expérience migratoire de sa mère Julia et de sa fratrie sont le fruit d'entretiens réalisés en Galice, en Catalogne et en France pour un travail de thèse sur les femmes migrantes galiciennes (en cours).

20.

Profitant de la générosité du gouvernement français et bénéficiant des premières politiques d'aide au retour des immigrés dans leur pays en 1978 contre la dévolution de leur passeport et l'engagement qu'ils ne reviendront pas, ils acquerront un « hostal » au cœur de Barcelone. Julia et José divorceront en 1978 et Julia conservera l'hostal qu'elle dirigera jusqu'à sa retraite prise tardivement, à 74 ans, en 2002. Elle passera les premiers mois de sa retraite au bord de la mer où elle avait acquis un petit appartement, mais seule et ne connaissant personne, elle décidera de retourner à Fruxil où elle a hérité d'une grange près de la ferme de ses parents. Elle y fera construire une petite maison où elle passera près de 15 ans.

21.

Les historiens des migrations ibériques expliquent les afflux massifs de migrants durant les années connues comme « les 30 glorieuses », par les besoins de la France comme de la plupart des pays européens développés d'une main-d'œuvre qu'ils accueillirent généreusement jusqu'à la fin des années 1970 et les premières lois dites « d'aide au retour ».

22.

Dans les années 1970 environ $10 \%$ des enfants d'ouvriers accédaient au second cycle d'études supérieures en France, et il y avait 6 à $7 \%$ d'étudiants étrangers. Pour les enfants d'ouvriers immigrés espagnols, moins de $1 \%$ d'entre eux terminaient le premier cycle (source: INSEE et Prost, Cyterman, 2010).

23.

Julia développe l'exemple d'un container à ordures ménagères qu'elle a réussi à promouvoir auprès des habitants du village qui continuaient, dans les années 2000, à évacuer leurs déchets dans la nature, et comment elle réussit à obtenir auprès des services de la mairie que le village soit équipé et desservi par les services municipaux.

24.

Site internet : <http://www.museodopobo.gal/web/museo.php>, consulté le 2 juin 2020.

25.

Vidéo disponible sur YouTube : https://www.youtube.com/watch ?v=o80lmpvgrc4, consulté le 4 janvier 2018.

26.

En dehors du contrôle des personnes, qui est l'obsession des dictateurs, l'objectif premier des accords bilatéraux que Franco signa avec des pays européens était de contrôler aussi les flux monétaires. 


\section{RÉSUMÉS}

En revenant sur le parcours migratoire d'une femme au sein d'une famille espagnole originaire de Galice immigrée en France et à travers le témoignage de sa fille, Amélie, qui tire un bilan négatif de la migration, cet article se propose, après avoir analysé les causes politiques et historiques qui ont produit deux types de migrations, l'exil politique et les émigrations "économiques", de comprendre et de montrer ce que les migrations font à la famille et comment les structures familiales survivent ou non à l'épreuve de la séparation dans le cas de parcours migratoires complexes ou comment des formes de «famille à distance » se recréent et survivent ou non aux ruptures et aux recommencements.

This article is the fruit of the observation of a Galician native family and more specifically a woman in particular in it, Julia, who emigrated from Galicia to Catalonia in the 40's just after the Spanish war, and later from Catalonia to France in the 60's. Our first source was Amalia's testimony, Julia's daughter as she makes a very negative balance of her mother's emigration. Our purpose is to see and understand the impact of forced migrations such as political exile or "economical emigrations" as a consequence of the major conflict of the 20th century in Spain, the Spanish civil war, produced on the family, for the persons but also how it transformed the social structure of the family. How the individuals deal -or not- with migration's consequences on familiar affective links and how does the family as a structure deal with them.

A través de la historia de una familia española de Galicia inmigrante en Francia en los años 1960 y mas precisamente de una mujer, Julia, en el seno de esta familia este articulo propone observar y reflexionar sobre el impacto en los sentimientos familiares y sobre la propia estructura de la familia que sigue produciendo el acontecimiento histórico y político de mayor magnitud del siglo XX en España, la guerra civil, al provocar movimientos forzados de población, se trate del exilio político o des las emigraciones "económicas". Nuestra primera fuente fue el testimonio de su hija, Amalia, que hace un balance muy negativo de la migración. Como se dislocaron y se reconstruyeron o no, las familias emigrantes, como resulta afectada la estructura de la familia reinventándose en modelos familiares alternativos, transnacionales, "familias en la distancia", para sobrevivir a las rupturas y nuevos inicios que supone la migración.

\section{INDEX}

Mots-clés : causes des migrations, famille, trajectoire migratoire, femmes

Index géographique : France, Espagne

\section{AUTEUR}

\section{EMMA RUBIO-MILET}

Université Paris-Est Marne-la-Vallée

emma.rubio@u-pem.fr 